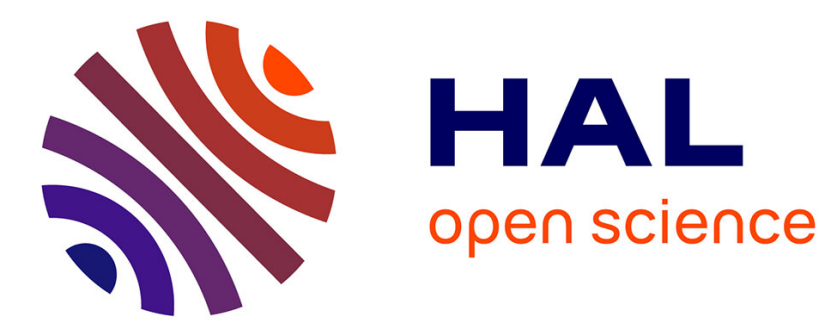

\title{
Comportement et durée de vie en fatigue isotherme de l'acier X 38 CrMoV 5
}

Denis Delagnes, Farhad Rezai-Aria, Christophe Levaillant, André Grellier

\section{To cite this version:}

Denis Delagnes, Farhad Rezai-Aria, Christophe Levaillant, André Grellier. Comportement et durée de vie en fatigue isotherme de l'acier X 38 CrMoV 5. Matériaux \& Techniques, 1999, n¹-2, p. 39-50. 10.1051/mattech/199987010039 . hal-01851452

\section{HAL Id: hal-01851452 \\ https://hal.science/hal-01851452}

Submitted on 6 Nov 2019

HAL is a multi-disciplinary open access archive for the deposit and dissemination of scientific research documents, whether they are published or not. The documents may come from teaching and research institutions in France or abroad, or from public or private research centers.
L'archive ouverte pluridisciplinaire HAL, est destinée au dépôt et à la diffusion de documents scientifiques de niveau recherche, publiés ou non, émanant des établissements d'enseignement et de recherche français ou étrangers, des laboratoires publics ou privés. 


\title{
COMPORTEMENT ET DURÉE DE VIE EN FATIGUE ISOTHERME DE L'ACIER X 38 CrMoV 5
}

\author{
D. Delagnes *, F. Rezaï-Aria *, C. Levaillant *, A. Grellier** \\ * Ecole des Mines d'Albi Carmaux, Campus Jarlard, Albi \\ ** Aubert \& Duval, Aciérie des Ancizes, Les Ancizes
}

\section{ISOTHERMAL FATIGUE BEHAVIOUR AND LIFETIME}

OF A $5 \%$ Cr HOT WORK TOOL STEEL

ABSTRACT: Dies and molds are subjected to severe mechanical and thermal loads during injection or forging operations. As fatigue is one of the most important cause of tool steels damage, we studied isothermal (between $200^{\circ} \mathrm{C}$ and $600^{\circ} \mathrm{C}$ ) fatigue behaviour and damage of two grades (SMV3 and ADC3) of a tempered martensitic 5\% Cr steel. More than 100 fatigue tests were performed in order to analyse mechanical loading, temperature and initial hardness influences. We observed a softening during all lifetime which is typical of tempered martensitic steels. Softening amplitudes are increasing with increasing total strain amplitude, and decreasing with increasing temperature (till $550^{\circ} \mathrm{C}$ ) and hardness (between $42 \mathrm{HRC}$ and $50 \mathrm{HRC}$ ).

Influences of temperature and hardness are limited when observed through a Manson-Coffin diagram. Conversely, increasing test temperature or decreasing hardness leads to a strong reduction of lifetime (factor 1000 for a 8 HRC decrease!) when results are plotted in a Basquin diagram. Interrupted tests showed that crack initiation controls lifetime for a number of cycles to failure around $10^{5}$ cycles. Three sites of crack initiation were identified and discussed:

- non metallic inclusions $\left(200^{\circ} \mathrm{C}-400^{\circ} \mathrm{C}\right)$;

- lath boundary $\left(200^{\circ} \mathrm{C}-400^{\circ} \mathrm{C}\right)$;

- prior austenitic grain boundary $\left(500^{\circ} \mathrm{C}-600^{\circ} \mathrm{C}\right)$.

Conférence présentée au colloque sur les "Aciers pour moules et outils" organisé les 27 et 28 mai 1998 à l'Ecole des Mines d'Albi par le Cercle d'Etude des Métaux. es aciers à $5 \%$ de chrome ont notamment été développés pour les outillages fonctionnant à des températures élevées. Les applications dans ce domaine sont nombreuses: moules d'injection sous pression d'alliages légers, matrices de forge, poinçons, mandrins, outillages d'extrusion, lames de cisaille, filières, etc.

Les matrices et les moules sont soumis à des sollicitations mécaniques et thermiques sévères au cours de l'injection ou du forgeage. La durée de vie limitée de ces outils a des conséquences économiques primordiales puisque le coût de l'outillage intervient pour 10 à $15 \%$ dans le prix de revient d'une pièce [1].

Cette étude s'inscrit dans une optique de caractérisation des propriétés en fatigue isotherme des aciers à outils. Son principal objectif concerne l'étude du comportement et des durées de vie en fatigue de l'acier martensitique revenu X 38 CrMoV $5^{(1)}$ autour de la transition fatigue oligocyclique-endurance. Les aciers martensitiques ont été relativement peu étudiés en fatigue. En effet, les données sont rares, probablement à cause de leur confidentialité pour les nuances récentes à applications industrielles. Cette étude répond donc à un réel manque. Par ailleurs, les durées de vie des outils s'étendent sur plusieurs ordres de grandeur. En effet, les poinçons pour travail à chaud peuvent être mis au rebut après quelques centaines de cycles alors qu'un moule du même alliage peut subir plusieurs centaines de milliers d'injections d'alliages de zinc [2]. Nous avons donc mené une campagne d'essais en déformation totale imposée

(1) Dénomination actuelle selon normes EN 10027-1 et NF A 35-590; anciennement Z 38 CDV 5 selon le fascicule de documentation NF A 02-005 (N.D.L.R.) 
dans une gamme de durées de vie comprises entre quelques milliers de cycles et quelques centaines de milliers de cycles de façon à nous rapprocher des durées de vie usuelles des moules d'injection d'aluminium.

Les principales caractéristiques du matériau et du plan d'expérience ainsi que les principaux équipements utilisés sont présentés dans la seconde partie.

La troisième partie traite d'un point de vue phénoménologique du comportement cyclique de l'acier X $38 \mathrm{CrMoV} 5$. Les résultats obtenus en fatigue oligocyclique à $300^{\circ} \mathrm{C}$ sont dans un premier temps examinés de façon à caractériser le comportement. Les influences de l'amplitude de la sollicitation, de la température d'essai et du niveau de dureté sur le comportement cyclique sont ensuite discutées. Ces trois paramètres ont en effet une grande influence sur la microstructure initiale ou sur son évolution au cours de l'essai.

La dernière partie concerne les influences de l'amplitude de la sollicitation, de la température d'essai et du niveau de dureté initial sur les durées de vie. Les différents sites d'amorçage observés en microscopie électronique à balayage sont présentés et discutés.

\section{Matériau étudié et programme expérimental}

\section{Acier X 38 CrMoV 5}

L'acier X 38 CrMoV 5 appartient à la classe des aciers à outils pour travail à chaud et fait partie de la famille des aciers à $5 \%$ de chrome et $0,4 \%$ de carbone. La composition des deux nuances étudiées (SMV3 et ADC3) qui différent essentiellement par leur teneur en silicium et en impuretés, est reportée dans le tableau I. Les traitements thermiques appliqués sont identiques sur les deux nuances. Après un traitement de recuit

Tableau I - Composition chimique des coulées étudiées (\% en poids). Table I - Chemical composition of investigated steels (weight \%).

\begin{tabular}{|l|l|c|c|c|c|c|c|c|}
\hline & \multicolumn{1}{|c}{$\mathrm{C}$} & $\mathrm{Si}$ & $\mathrm{Mn}$ & $\mathrm{Ni}$ & $\mathrm{Cr}$ & $\mathrm{Mo}$ & $\mathrm{V}$ & $\mathrm{Fe}$ \\
\hline Nuance SMV3 & 0,4 & 0,92 & 0,49 & 0,20 & 5,05 & 1,25 & 0,47 & bal. \\
\hline Nuance ADC3 & 0,361 & 0,35 & 0,36 & 0,06 & 5,06 & 1,25 & 0,49 & bal. \\
\hline
\end{tabular}

Photo 1 - Structure en lattes de la nuance SMV3 (attaque nital 3\%, MEB). Photo I - Lath martensitic structure of SMV3 grade (3\% nital etch, SEM).

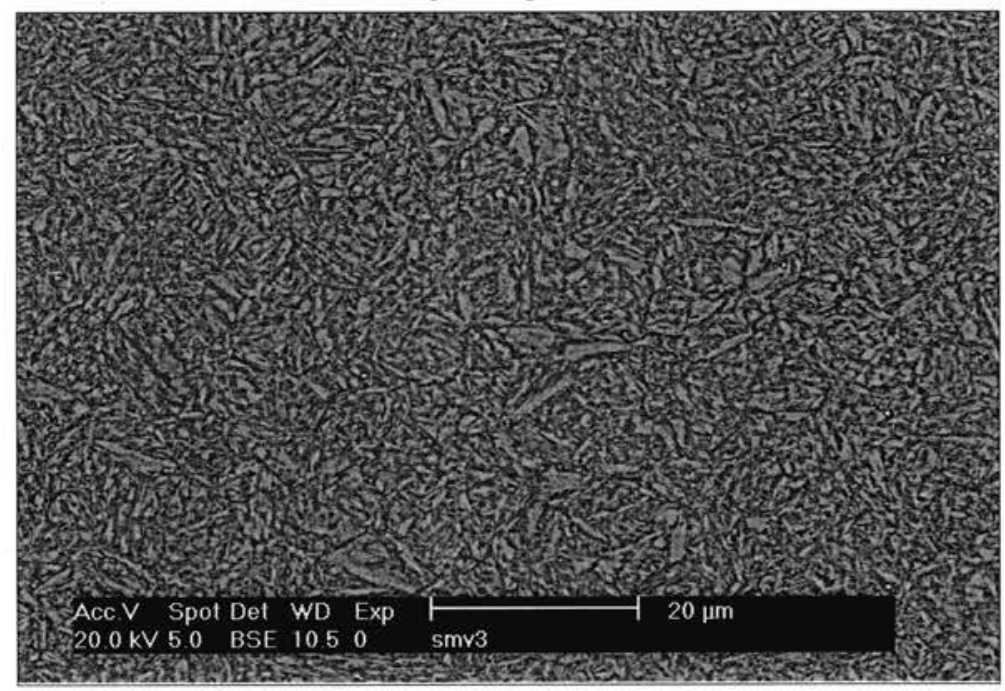

qui nécessite des cycles thermiques spécifiques qui font partie du savoir-faire de chaque aciériste [3], les ébauches des éprouvettes sont usinées. L'austénitisation s'effectue à $990^{\circ} \mathrm{C}$ pendant 1 heure. Elle est suivie d'une trempe à l'air calme; le temps nécessaire pour refroidir l'ébauche depuis la température d'austénitisation jusqu'à une température inférieure à $100^{\circ} \mathrm{C}$ est de 40 minutes. Le diagramme TRC d'un acier X 38 CrMoV 5 montre que la trempe conduit à une structure qui n'est pas complètement martensitique [4]. Le terme d'acier bainitomartensitique est donc plus adapté. Enfin, un double revenu est réalisé, le premier à $550^{\circ} \mathrm{C}$ pendant deux heures et le second entre $590^{\circ} \mathrm{C}$ et $625^{\circ} \mathrm{C}$ suivant le niveau de dureté désiré. Les éprouvettes de fatigue sont ensuite usinées.

La microstructure obtenue est une microstructure en lattes de taille hétérogène variant entre $0,1 \mu \mathrm{m}$ et $2 \mu \mathrm{m}$ en largeur et entre $0,5 \mu \mathrm{m}$ et $15 \mu \mathrm{m}$ en longueur (photo 1). Une attaque électrolytique à l'acide chromique permet de révéler les joints de grain de la structure austénitique initiale (photo 2). Le diamètre moyen des grains est compris entre 10 et $14 \mu \mathrm{m}$ pour 50 grains analysés suivant la nuance d'acier X $38 \mathrm{CrMoV} 5$ observée. La taille de grain est de type 10/12 avec quelques plages de type 7/8 suivant la norme NF A 04-102 [5].

\section{Plan d'expérience}

Le plan d'expérience est divisé en deux parties (cf. tableau II): - la caractérisation d'un état de référence, qui représente la partie la plus volumineuse du plan;

- l'étude de l'influence du niveau de dureté.

Notre choix concernant l'état de référence a été guidé par la nécessaire représentativité de cet état au niveau industriel. Les deux nuances étudiées ont donc subi un traitement thermique pour obtenir un niveau de dureté de $42 \mathrm{HRC}$, niveau équivalent à celui des moules d'injection pour pièces de grande taille (carter de boite de vitesse par exemple) [6].

Les traitements thermiques effectués sur les aciers à outils varient suivant leurs utilisations et conduisent à des microstructures différentes responsables des propriétés mécaniques spécifiques. La dernière étape du traitement thermique (deuxième revenu) est modifiée de façon à obtenir des niveaux de dureté compris entre $42 \mathrm{HRC}$ et 50 HRC.
Photo 2 - Joints de grain de la structure austénitique initiale (nuance ADC3).

Photo 2 - Prior austenitic grain boundaries (ADC3 grade).

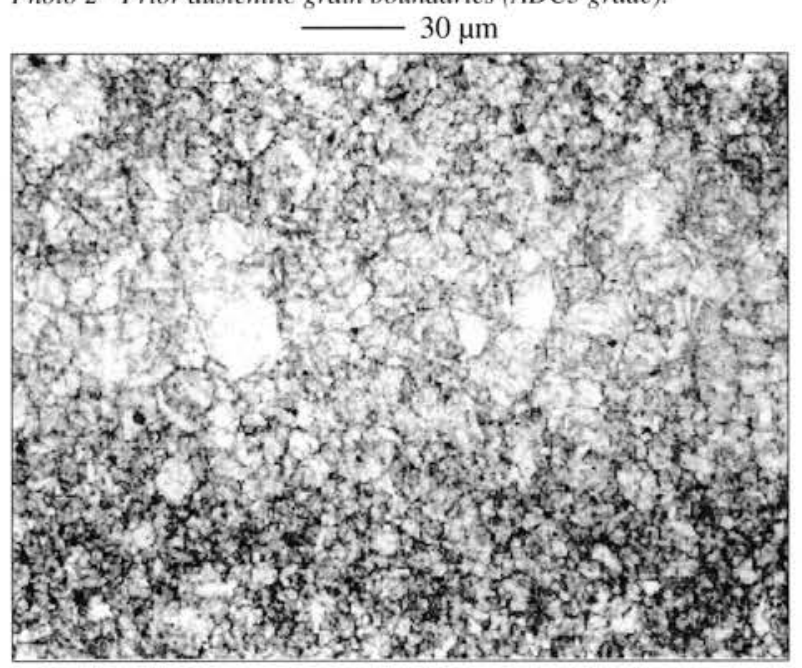




\begin{tabular}{|c|c|c|c|c|c|}
\hline & Nuance(s) & $\begin{array}{c}\text { Nombre de } \\
\text { températures } \\
\text { d'essai }\end{array}$ & $\begin{array}{c}\text { Nombre } \\
\text { d'éprouvettes } \\
\text { par température }\end{array}$ & $\begin{array}{c}\text { Niveau } \\
\text { de dureté } \\
\text { (HRC) }\end{array}$ & $\begin{array}{c}\text { Nombre } \\
\text { total } \\
\text { d'éprouvettes }\end{array}$ \\
\hline $\begin{array}{c}\text { ETAT } \\
\text { DE } \\
\text { RÉFÉRENCE }\end{array}$ & ADV3 & 6 & 4 à 8 & 42 & 80 \\
\hline $\begin{array}{c}\text { TRAITEMENT } \\
\text { THERMIQUE I }\end{array}$ & ADC3 & 4 & 4 à 7 & 47 & 20 \\
\hline $\begin{array}{c}\text { TRAITEMENT } \\
\text { THERMIQUE II }\end{array}$ & ADC3 & 2 & 5 & 44 & 10 \\
\hline $\begin{array}{c}\text { TRAITEMENT } \\
\text { THERMIQUE III }\end{array}$ & ADC3 & 2 & 5 & 50 & 10 \\
\hline NOMBRE TOTAL D'ESSAIS & & & & 120 \\
\hline
\end{tabular}

Tableau II - Tableau synoptique du plan d'expérience. Table Il - Experimental schedule.

Tableau III - Gamme de polissage.

Table III - Polishing range.

\begin{tabular}{|c|c|}
\hline Type de polissage & Durée \\
\hline papier abrasif 320 & $1 \mathrm{~h} 00$ \\
\hline papier abrasif 600 & $1 \mathrm{~h} 00$ \\
\hline papier abrasif 1200 & $1 \mathrm{~h} 30$ \\
\hline suspension diamant $6 \mu \mathrm{m}$ & $2 \mathrm{~h} 00$ \\
\hline suspension diamant $3 \mu \mathrm{m}$ & $3 \mathrm{~h} 00$ \\
\hline suspension diamant $1 \mu \mathrm{m}$ & $3 \mathrm{~h} 00$ \\
\hline
\end{tabular}

\section{Techniques expérimentales}

Une machine d'essai Schenck Hydropuls série 56 de force nominale $250 \mathrm{kN}$ a été utilisée pour la réalisation du plan d'expérience. La mesure de la déformation est assurée par un extensomètre longitudinal adapté aux essais à haute température et à nos conditions expérimentales. L'extensomètre dont la base de mesure est $10 \mathrm{~mm}$ (valeur optimisée pour éviter des gradients thermiques trop élevés), est placé dans un boîtier refroidi par eau à l'extérieur du four (photo 3). À l'aide de deux pinces de serrage, l'extensomètre est rendu solidaire d'un système mécanique sur lequel sont fixées deux tiges d'alumine. Ces tiges d'alumine, coniques à leurs extrémités, entrent dans le four et viennent palper l'éprouvette.

Enfin, le chauffage de l'éprouvette est assuré par un four à résistance d'une puissance de $1 \mathrm{~kW}$. Le gradient de température sur la partie calibrée est au maximum de $6^{\circ} \mathrm{C}\left(\right.$ à $\left.600^{\circ} \mathrm{C}\right)$.

Les températures d'essai sont comprises entre $200^{\circ} \mathrm{C}$ et $600^{\circ} \mathrm{C}$, cette dernière température étant très proche de celle du second revenu; elle est supérieure à la température d'utilisation conseillée du matériau.

Les conditions d'essai (sollicitations, fréquence) doivent être compatibles avec les moyens disponibles, notamment en ce qui concerne la fréquence d'échantillonnage et le stockage des données. Le temps consacré à un essai (dont le nombre de cycles à rupture est de 100000) est de deux jours, le montage, le chauffage, le refroidissement, le démontage et le traitement

Photo 3 - Vue du dispositif expérimental avant essai : éprouvette, extensomètre et four.

Photo 3 - Experimental set-up view before testing : specimen, extensometer and furnace.

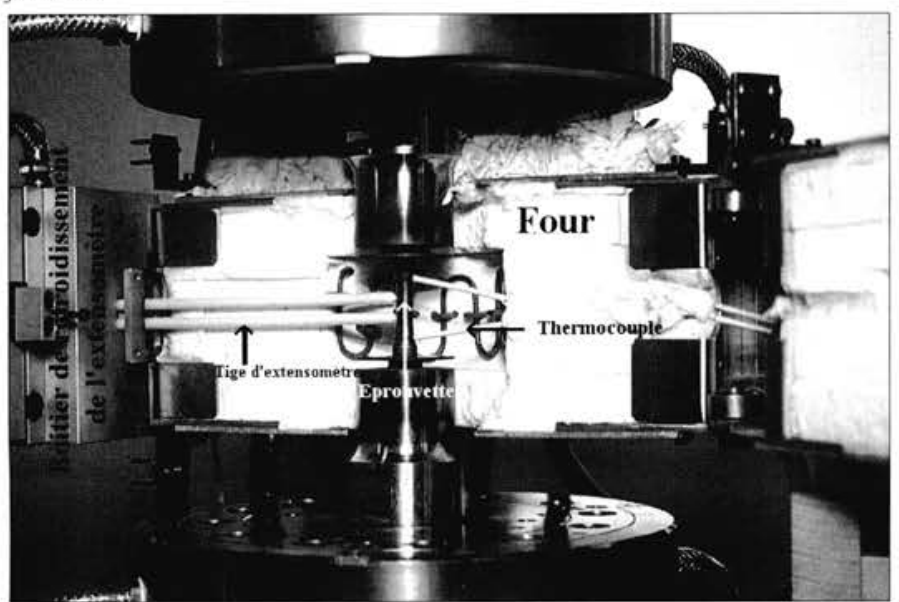

de données étant compris. Une fréquence d'essai de 1 Hertz, compatible d'une part avec la fréquence d'échantillonnage et d'autre part avec la nécessité d'obtenir des durées d'essai raisonnables, a été fixée.

Afin de réduire les dispersions sur les durées de vie, la préparation de l'état de surface a été particulièrement soignée de façon à assurer une reproductibilité parfaite d'une éprouvette à l'autre. Un polissage automatique à la pâte diamantée dans le sens long a été réalisé. La gamme de polissage est indiquée dans le tableau III.

\section{Résultats et discussion}

Comportement en fatigue isotherme de l'acier X 38 CrMoV 5

La figure 1 représente la demi-amplitude de contrainte en fonction du nombre de cycles à $300^{\circ} \mathrm{C}$ pour une amplitude de déformation totale de $1,4 \%$. La courbe peut être décomposée en trois zones:

- zone A: stade de déconsolidation forte concentré sur les premières centaines de cycles;

- zone B: stade de pseudo-stabilité ou de déconsolidation faible;

- zone C: stade de perte de pseudo-stabilité due à la présence d'une ou de plusieurs fissures macroscopiques dans la partie utile de l'éprouvette.

Fig. 1 - Comportement cyclique de la nuance SMV3 à basse température. Figure 1 - SMV3 grade cyclic behaviour at low temperature.

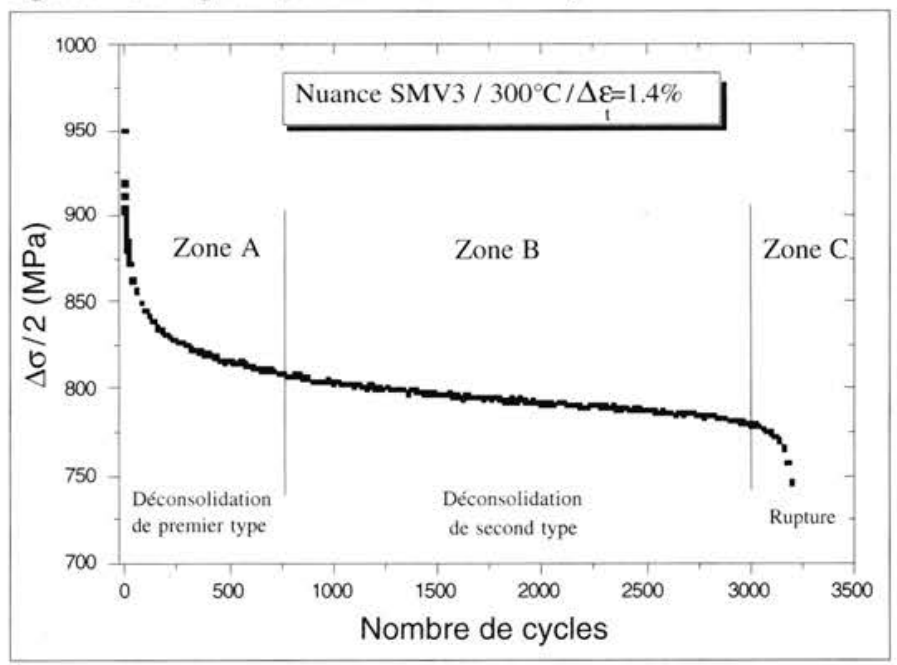


À basse température et en fatigue oligocyclique, l'acier X 38 CrMoV 5 présente une déconsolidation (adoucissement) cyclique continue jusqu'à la rupture sans atteindre de stabilisation. Ce comportement est caractéristique des aciers martensitiques ou bainitiques revenus [7-20]. Des observations en microscopie électronique en transmission sur des éprouvettes provenant d'essais interrompus ont permis de mettre en évidence les principaux mécanismes responsables de la déconsolidation cyclique. Il s'agit de l'évolution au cours du cyclage de la structure de dislocation et des précipités.

Au niveau de la structure de dislocation, la densité initiale de dislocations est élevée $\left(\sim 10^{11} \mathrm{~cm}^{-2}\right)$, ces dislocations étant réparties de façon relativement homogène bien que, d'un point de vue microscopique, l'enchevêtrement de dislocations apparaisse plus dense aux joints de grain, aux frontières des phases et autour des précipités. Après quelques cycles, l'enchevêtrement est graduellement rompu et il se développe alors des cellules de dislocations dont la paroi est large et incomplète en début d'essai pour devenir étroite et bien définie lorsque la stabilité ou la pseudo-stabilité est atteinte. La densité de dislocation réduite à l'intérieur des cellules favorise un mouvement plus libre des dislocations et donc une augmentation de la déformation plastique. Ce mécanisme fait l'unanimité. Aux faibles amplitudes de sollicitation, les cellules sont beaucoup moins nettes et restent encore incomplètes, ce qui pourrait entraîner l'absence de saturation cyclique.

Par ailleurs, l'annihilation des dislocations à l'aide des mécanismes de glissement dévié ou de montée de dislocations aux températures élevées peuvent également contribuer à l'adoucissement cyclique.

Aux températures plus élevées, l'augmentation de la taille des précipités, qui conduit à une diminution de leur densité, a pour effets d'une part de faire perdre une partie de la résistance obtenue par une fine précipitation lors du revenu et, d'autre part, d'augmenter la mobilité des dislocations, d'où une déconsolidation cyclique accrue. Par ailleurs, la morphologie en bâtonnet très allongé (témoignage d'une cohérence cristallographique avec la matrice) est modifiée pour obtenir une forme plus équiaxe, plus globulaire. Les carbures susceptibles de globulariser sont les carbures de molybdène, de chrome et surtout de fer [21].

Le premier régime témoigne d'une perte assez considérable de l'amplitude de contrainte en quelques centaines de cycles ( $100 \mathrm{MPa}$ sur les 100 premiers cycles). La déconsolidation constatée en zone A est appelée déconsolidation de premier type. Le deuxième régime correspond à une déconsolidation linéaire. La transition entre les deux régimes n'est pas franche. Afin d'évaluer $N_{t r}$, nombre de cycles nécessaire à la transition entre le stade de déconsolidation de premier type et le stade de déconsolidation linéaire, le critère adopté est indiqué sur la figure 2. Les deux paramètres $h$ et $i$ de la loi $\Delta \sigma / 2=h N+i$ sont déterminés par une régression linéaire à l'aide des données sélectionnées lorsque le premier régime est dépassé sans ambiguïté. La droite ainsi déterminée est prolongée vers les faibles nombres de cycle et un écart de $3 \mathrm{MPa}$ (supérieur aux incertitudes de mesure constatées expérimentalement) par rapport à cette droite définit $N_{t r}$. En prolongeant cette même droite vers les nombres de cycles élevés, un même critère est appliqué de façon à définir $N^{*}$ qui correspond à la durée de vie. La déconsolidation qui correspond à la zone $\mathrm{B}$ est appelée déconsolidation de deuxième type ou linéaire. Des essais interrompus ont

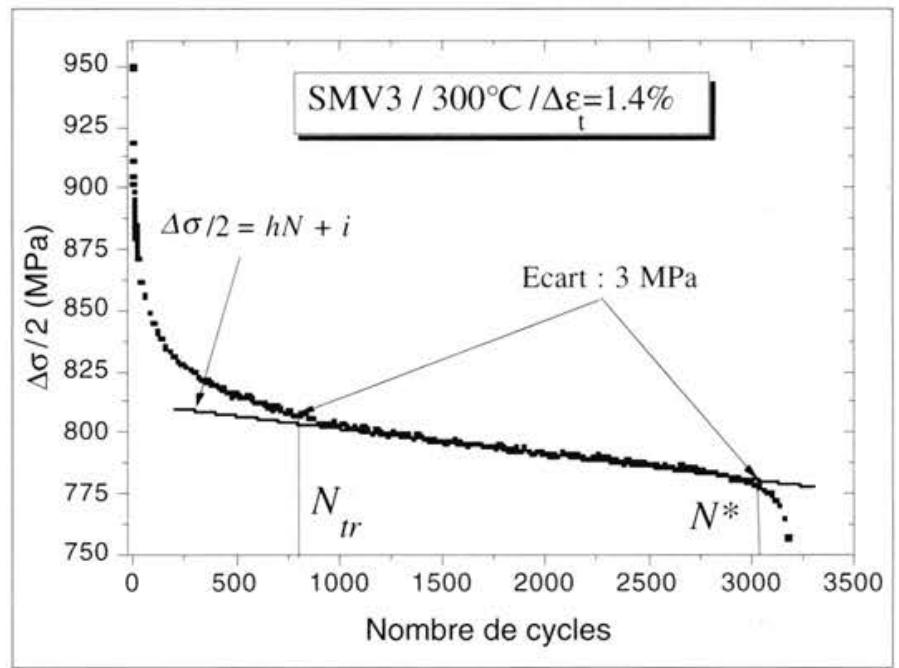

Fig. 2 - Définition des cycles Ntr et $N^{*}$.

Fig. 2 - Definition of cycles Ntr and $N^{*}$.

permis de relever la taille des fissures à l'aide de la technique des répliques. Nous avons pu montrer que la déconsolidation linéaire n'est pas due à la propagation de fissures macroscopiques mais est bien de type "comportementale" [22]. Au cycle $N^{*}$, la profondeur de fissure est de l'ordre de $200 \mu \mathrm{m}$. La perte d'amplitude de contrainte constatée en zone C n'est pas seulement la conséquence d'une déconsolidation cyclique due aux mécanismes précédemment décrits. En effet, la présence d'une ou de plusieurs fissures macroscopiques, et donc la perte de section résistante, a pour effet de réduire l'amplitude de contrainte nécessaire à la déformation de la partie utile de l'éprouvette. L'essai est interrompu lorsque la contrainte maximale chute de $10 \%$; la profondeur de la fissure principale est alors supérieure à $1 \mathrm{~mm}$.

L'amplitude de déconsolidation totale $D$ est décomposée en amplitude de déconsolidation de premier type $\left(1<N<N_{t r}\right)$ appelée $D_{l}$ et de second type $\left(N_{t r}<N<N^{*}\right)$ appelée $D_{2}$, avec:

$$
\begin{aligned}
& D_{1}=\left(\frac{\Delta \sigma}{2}\right)_{N=1}-\left(\frac{\Delta \sigma}{2}\right)_{N=N_{t r}} \\
& D_{2}=\left(\frac{\Delta \sigma}{2}\right)_{N=N_{t r}}-\left(\frac{\Delta \sigma}{2}\right)_{N=N^{*}}
\end{aligned}
$$

et

$$
D=D_{1}+D_{2}
$$

Dans la gamme explorée, une diminution de l'amplitude de déformation totale conduit à une plus grande stabilité cyclique du matériau puisque l'amplitude de déconsolidation totale chute rapidement (fig. 3). D'autre part, nous constatons une tendance à la saturation des amplitudes de déconsolidation de premier et de second type aux amplitudes de déformation totale ou plastique (prises à $N * / 2$ ) élevées (cf. fig. 4). À $300^{\circ} \mathrm{C}$, les amplitudes de déconsolidation totale atteignent $170 \mathrm{MPa}$, soit environ $20 \%$ de l'amplitude de contrainte au premier cycle, ce qui est considérable.

\section{Influence de la température d'essai}

Les stades de déconsolidations de premier type et linéaire sont constatés jusqu'à $550^{\circ} \mathrm{C}$ inclus pour toutes les sollicitations imposées (fig. 5).

Les amplitudes de déconsolidation totale ne subissent pas de variations significatives pour une amplitude de déformation 


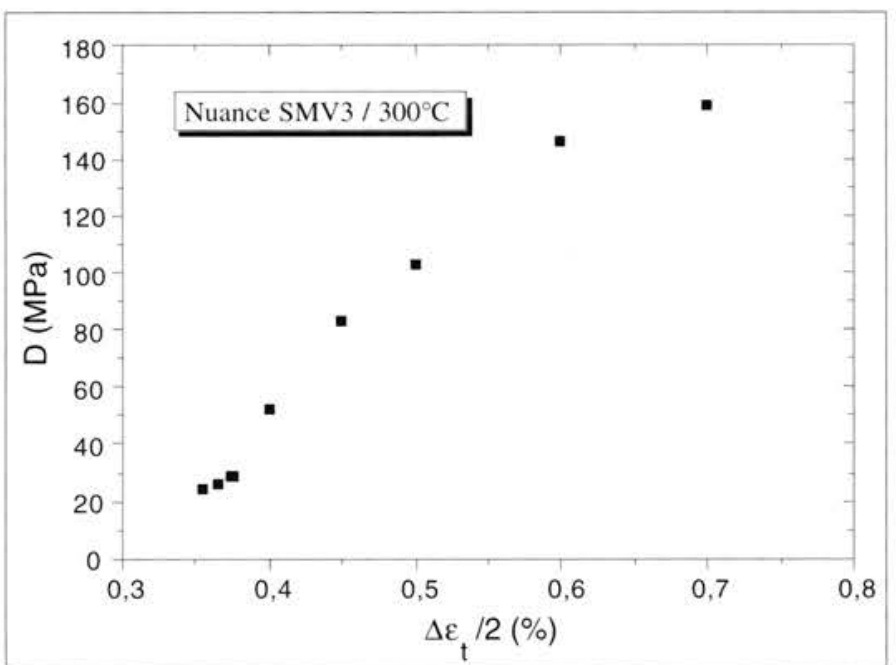

Fig. 3 - Amplitude de déconsolidation totale (Nuance SMV3 à $300^{\circ} \mathrm{C}$ ).

Fig. 3 - Total softening amplitude (SMV3 grade at $\left.300^{\circ} \mathrm{C}\right)$.

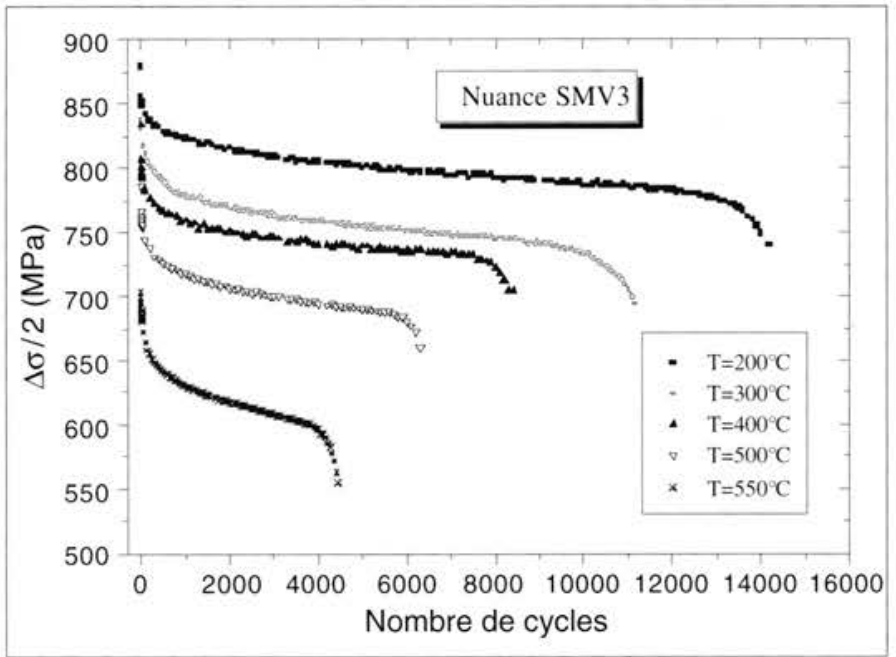

Fig. 5 - Influence de la température sur le comportement cyclique (nuance SMV3; $\Delta \varepsilon_{1}=1 \%$ ).

Fig. 5 - Temperature influence on cyclic behaviour (SMV3 grade $\left./ \Delta \varepsilon_{t}=I \%\right)$.

totale donnée. Cependant, l'amplitude de déformation plastique augmentant avec la température, les amplitudes de déconsolidation diminuent à amplitude de déformation plastique équivalente (fig. 6). L'acier X $38 \mathrm{CrMoV} 5$ est donc plus stable aux températures élevées. Toutefois, à $600^{\circ} \mathrm{C}$, l'amplitude de déconsolidation linéaire a tendance à augmenter sensiblement. À cette température proche du second revenu, une modification de la taille ou de la nature des précipités au cours du cyclage peut en être la cause $[8,20,21,23]$.

\section{Influence du niveau de dureté initial}

La limite d'élasticité étant plus élevée à niveau de dureté croissante, les déformations plastiques, pour une même amplitude de déformation totale imposée, sont donc plus faibles, si bien que les déconsolidations sont également plus faibles (fig. 7). De même, la figure 8 montre que pour une même déformation plastique, la déconsolidation cyclique est légèrement plus faible pour les niveaux de dureté élevés. L'acier martensitique revenu $X 38$ CrMoV 5 a tendance à devenir plus stable lorsque le niveau de dureté augmente. Cette meilleure stabilité cyclique peut être associée à une densité de dislocations et de carbures plus importante qui font obstacle au mouvement des dislocations.

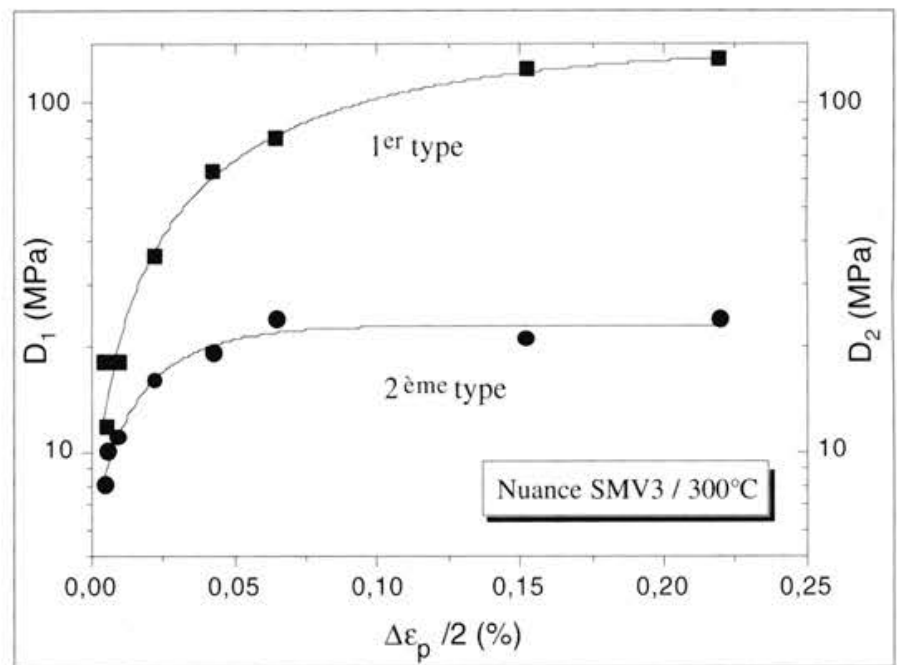

Fig. 4 - Saturation des amplitudes de déconsolidation (nuance SMV3 à $300^{\circ} \mathrm{C}$ ). Fig. 4 - Softening amplitudes saturation (SMV3 grade at $300^{\circ} \mathrm{C}$ ).

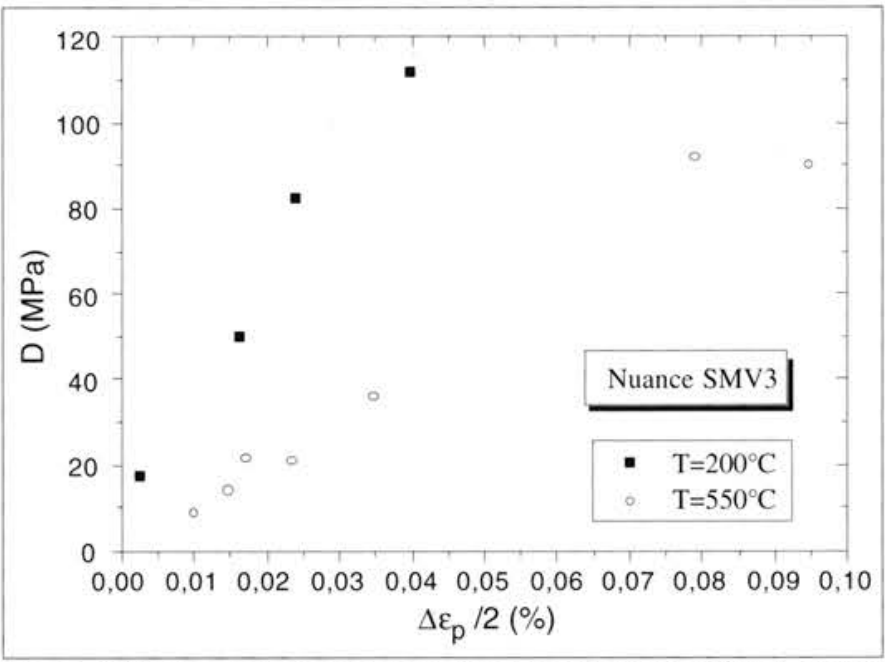

Fig. 6 - Influence de la température sur l'amplitude de déconsolidation totale en fonction de $\Delta \varepsilon_{\mathrm{p}} / 2$ (à $\left.\mathrm{N} * / 2\right)$.

Fig. 6 - Temperature influence on total softening amplitude versus $\Delta \varepsilon_{p} / 2$ (at $N * / 2$ ).

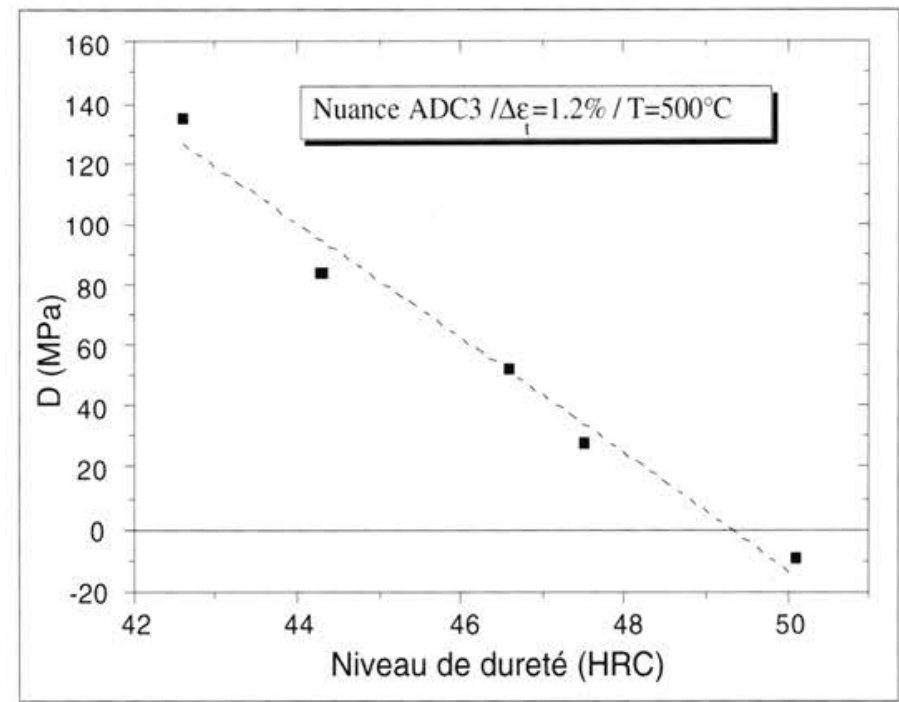

Fig. 7 - Influence du niveau de dureté sur l'amplitude de déconsolidation totale (notez la consolidation à $\mathbf{5 0}$ HRC).

Fig. 7 - Hardness influence on total softening amplitude (note the cyclic hardening at $50 \mathrm{HRC}$ ). 


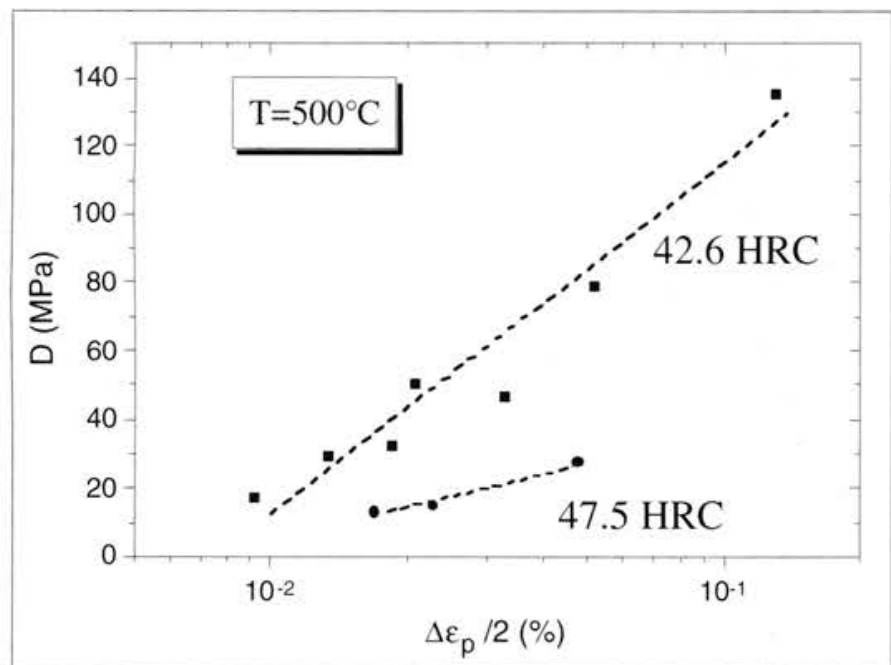

Fig. 8 - Influence du niveau de dureté sur l'amplitude de déconsolidation totale en fonction de $\Delta \varepsilon_{\mathrm{p}} / 2$ à $\mathrm{N} * / 2$ (nuance ADC3).

Fig. 8 - Hardness influence on total softening amplitude versus $\Delta \varepsilon_{p} / 2$ at $N^{*} / 2$ (ADC3 grade).

En décomposant la déconsolidation totale en déconsolidation de premier et de second type, le niveau de dureté a peu d'influence sur la déconsolidation linéaire alors que l'amplitude de déconsolidation de premier type diminue très fortement pour devenir négative à $50 \mathrm{HRC}$ (fig. 9). Nous observons donc une consolidation cyclique à $50 \mathrm{HRC}$ pour les faibles amplitudes de sollicitation. Cette consolidation est généralement associée à une hétérogénéité de la microstructure. En effet, elle est expliquée par une accommodation préférentielle des zones ferritiques ou austénitiques dans la littérature [14-16, 18, 19, 24]. Nous constatons également une hétérogénéité de structure. En effet, quelques zones non aiguillées, pour lesquelles on peut supposer une densité de dislocations amoindrie, apparaissent après une attaque au réactif de Catella; cependant, la nature de ces zones n'a pas été formellement identifiée.

\section{Durées de vie}

\section{Influence de la température d'essai}

Nous avons relié les durées de vie obtenues aux amplitudes de déformation plastique et de contrainte (prises à demi-durée de vie) à l'aide des lois de Manson-Coffin et de Basquin:

$$
\begin{aligned}
\frac{\Delta \varepsilon_{p}}{2} & =\varepsilon_{f}^{\prime}\left(2 N^{*}\right)^{c} \\
\frac{\Delta \sigma}{2} & =C_{e}\left(N^{*}\right)^{p}
\end{aligned}
$$

où $c$ est l'exposant de ductilité en fatigue, $\varepsilon_{f}$ est le coefficient de ductilité en fatigue, $p$ est l'exposant de Basquin et $C_{e}$ est le coefficient de Basquin.

Des facteurs de dispersion sur les durées de vie de 2 pour les lois de Manson-Coffin et de 1,5 sur les lois de Basquin sont obtenus [22]. Les paramètres sont reportés dans les tableaux IV et $\mathrm{V}$.

Sur les diagrammes de Manson-Coffin, deux régimes peuvent être identifiés. Jusqu'à $550^{\circ} \mathrm{C}$, les droites de Manson-Coffin, compte tenu des dispersions constatées, ne semblent pas se différencier. Au-delà, une même amplitude de déformation plastique donne une durée de vie plus courte. À $600^{\circ} \mathrm{C}$, une diminution de durée de vie d'environ $60 \%$ par rapport à celle obtenue entre $200^{\circ} \mathrm{C}$ et $500^{\circ} \mathrm{C}$ est observée (fig. 10).

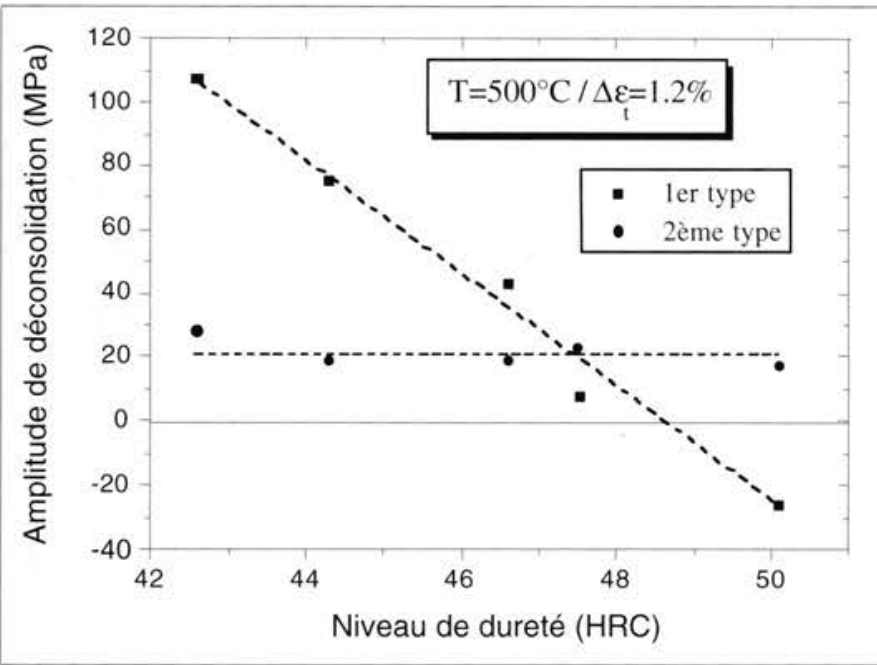

Fig. 9 - Influence du niveau de dureté sur les amplitudes de déconsolidation de premier et de second type (nuance ADC3).

Fig. 9 - Hardness influence on first and second type of softening amplitudes (ADC3 grade).

En ce qui concerne le diagramme de Basquin, l'influence de la température apparaît beaucoup plus nette. Pour une même amplitude de contrainte à demi-durée de vie, la température a une influence très sensible sur les durées de vie, cette influence augmentant considérablement pour des températures supérieures à $500^{\circ} \mathrm{C}$. La perte de durée de vie peut atteindre un facteur supérieur à 200 entre $500^{\circ} \mathrm{C}$ et $600^{\circ} \mathrm{C}$ (fig. 11 ).

\section{Influence du niveau de dureté}

L'influence du niveau de dureté initial sur les durées de vie est déterminée pour des températures comprises entre $300^{\circ} \mathrm{C}$ et $600^{\circ} \mathrm{C}$ et des niveaux de dureté compris entre 42 et $50 \mathrm{HRC}$ (les paramètres étant reportés dans les tableaux VI et VII).

Sur les diagrammes de Manson-Coffin, il paraît bien ambitieux de déduire une influence quantitative du niveau de dureté sur les durées de vie. En effet, plus le niveau de dureté est élevé, plus les amplitudes de déformation plastique deviennent faibles (et l'erreur importante) voire non mesurables. Toutefois, deux tendances se dégagent:

- à toutes températures, l'augmentation du niveau de dureté jusqu'à 46-47 HRC n'induit pas de modifications sensibles sur les droites de Manson-Coffin;

- Au-delà de 47 HRC, dans le diagramme de Manson-Coffin, la durée de vie diminue (fig. 12). Cet effet est légèrement plus prononcé pour les fortes durées de vie; par contre, il a tendance à s'estomper à $600^{\circ} \mathrm{C}$.

Tableau IV - Paramètres de la loi de Manson-Coffin pour la nuance SMV3. Table IV-Manson-Coffin parameters for the SMV3 grade.

\begin{tabular}{|c|c|c|c|c|c|c|}
\hline Température $\left({ }^{\circ} \mathrm{C}\right)$ & 200 & 300 & 400 & 500 & 550 & 600 \\
\hline$\varepsilon_{f}^{\prime}$ & 4652,5 & 2261,2 & 48,4 & 132,1 & 184,9 & 121,0 \\
\hline$c$ & $-1,15$ & $-1,06$ & $-0,71$ & $-0,80$ & $-0,85$ & $-0,86$ \\
\hline
\end{tabular}

Tableau V - Paramètres de la loi de Basquin pour la nuance SMV3. Table V-Basquin parameters for the SMV3 grade.

\begin{tabular}{|c|c|c|c|c|c|c|}
\hline Température $\left({ }^{\circ} \mathrm{C}\right)$ & 200 & 300 & 400 & 500 & 550 & 600 \\
\hline $\boldsymbol{C}_{e}$ & 1585 & 1366 & 1201 & 1094 & 965 & 1062 \\
\hline$p$ & $-0,074$ & $-0,068$ & $-0,052$ & $-0,052$ & $-0,057$ & $-0,086$ \\
\hline
\end{tabular}




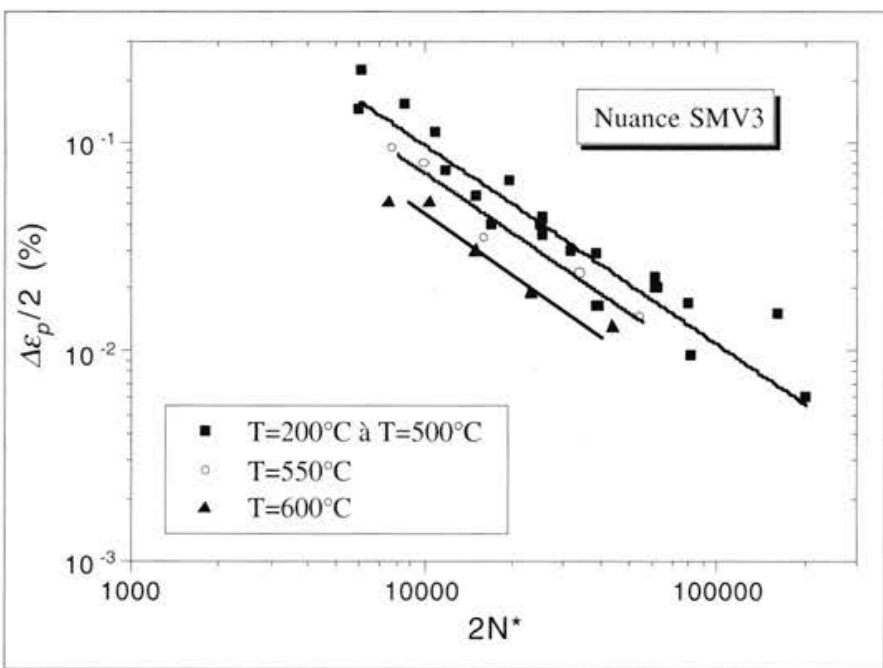

Fig. 10 - Influence de la température sur les durées de vie dans un diagramme de Manson-Coffin (nuance SMV3).

Fig. 10-Temperature influence on lifetimes in a Manson-Coffin diagram (SMV3 grade).

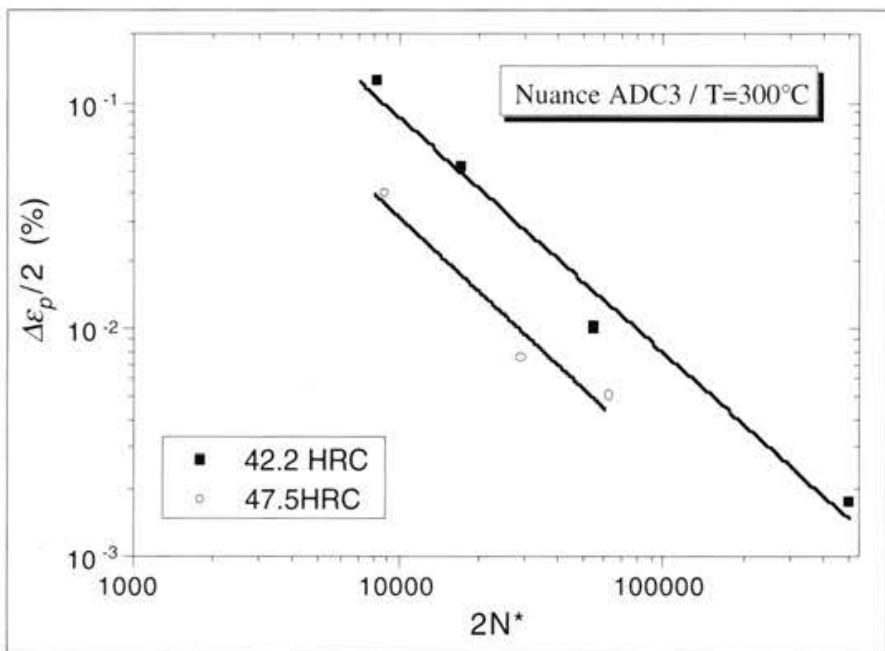

Fig. 12 - Diagramme de Manson-Coffin pour la nuance $\mathrm{ADC} 3$ à $300^{\circ} \mathrm{C}$ : effet du niveau de dureté.

Fig. 12 - Manson-Coffin diagram for the ADC3 grade at $300^{\circ} \mathrm{C}$ : hardness effect.

La figure 13 présente le diagramme de Manson-Coffin à $500^{\circ} \mathrm{C}$ pour différents niveaux de dureté avec des amplitudes de déformation plastique mesurées au premier cycle. Les différences ont totalement disparu. Il est donc possible d'attribuer

Tableau VI - Paramètres de la loi de Manson-Coffin pour la nuance ADC3. Table VI-Manson-Coffin parameters for the ADC3 grade.

\begin{tabular}{|c|c|c|c|c|c|c|c|}
\hline \multirow{2}{*}{$\begin{array}{c}\text { Tempé- } \\
\text { rature } \\
\left({ }^{\circ} \mathrm{C}\right)\end{array}$} & $\begin{array}{c}\text { Paramètres } \\
\text { de Manson- } \\
\text { Coffin }\end{array}$ & \multicolumn{6}{|c|}{ Niveau de dureté (HRC) } \\
\hline \multirow{2}{*}{300} & $\varepsilon_{f}^{\prime}$ & 1249,4 & - & - & - & 702,9 & - \\
\cline { 2 - 9 } & $c$ & $-1,04$ & - & - & - & $-1,09$ & - \\
\hline \multirow{2}{*}{500} & $\varepsilon_{f}^{\prime}$ & - & 6,4 & 5,7 & - & 2,9 & 5,4 \\
\cline { 2 - 9 } & $c$ & - & $-0,53$ & $-0,52$ & - & $-0,48$ & $-0,59$ \\
\hline \multirow{2}{*}{550} & $\varepsilon_{f}^{\prime}$ & 4,2 & - & 2,5 & 17,0 & 6,8 & 4,1 \\
\cline { 2 - 9 } & $c$ & $-0,48$ & - & $-0,43$ & $-0,64$ & $-0,55$ & $-0,56$ \\
\hline \multirow{2}{*}{600} & $\varepsilon_{f}^{\prime}$ & - & 6,4 & - & - & 261,0 & - \\
\hline & $c$ & - & $-0,56$ & - & - & $-0,99$ & - \\
\hline
\end{tabular}

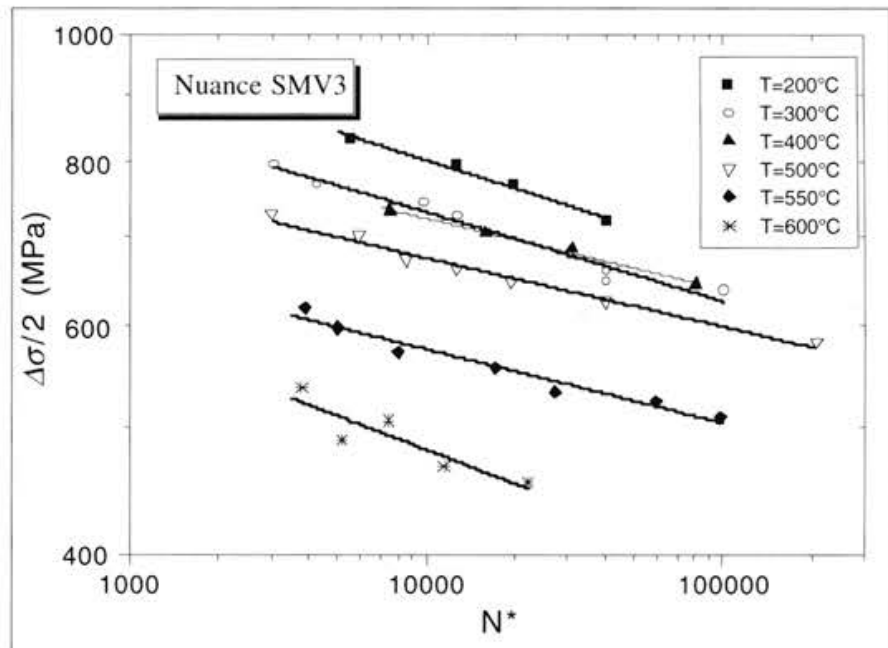

Fig. 11 - Influence de la température sur les durées de vie dans un diagramme de Basquin (nuance SMV3).

Fig. 11 - Temperature influence on lifetimes in a Basquin diagram (SMV3 grade).

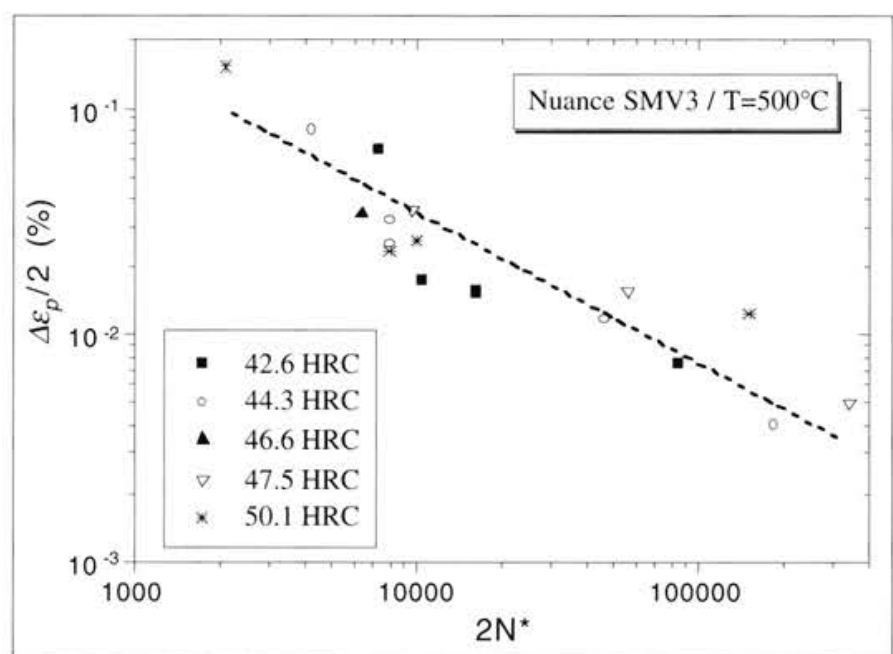

Fig. 13 - Diagramme de Manson-Coffin avec les amplitudes de déformation plastique mesurées au premier cycle.

Fig. 13-Manson-Coffin diagram with plastic strain amplitudes measured at the first cycle.

les différences constatées pour des amplitudes de déformation plastique prises à demi-durée de vie par une différence de comportement cyclique. En effet, l'acier X $38 \mathrm{CrMoV} 5$ possède une meilleure stabilité cyclique pour des niveaux de dureté

Tableau VII - Paramètres de la loi de Basquin pour la nuance ADC3. Table VII-Basquin parameters for the ADC3 grade.

\begin{tabular}{|c|c|c|c|c|c|c|c|}
\hline \multirow{2}{*}{$\begin{array}{c}\text { Tempé- } \\
\text { rature } \\
\left({ }^{\circ} \mathrm{C}\right)\end{array}$} & $\begin{array}{c}\text { Paramètres } \\
\text { de }\end{array}$ & \multicolumn{6}{|c|}{ Niveau de dureté (HRC) } \\
\cline { 3 - 8 } & Basquin & 42,2 & 42,6 & 44,3 & 46,6 & 47,5 & 50,1 \\
\hline \multirow{2}{*}{300} & $C_{e}$ & 1346 & 1367 & - & - & 1868 & - \\
\cline { 3 - 8 } & $p$ & $-0,061$ & $-0,063$ & - & - & $-0,077$ & - \\
\hline \multirow{2}{*}{500} & $C_{e}$ & - & 1045 & 1172 & 1281 & 1407 & 1526 \\
\cline { 2 - 9 } & $p$ & - & $-0,048$ & $-0,050$ & $-0,048$ & $-0,053$ & $-0,054$ \\
\hline \multirow{2}{*}{550} & $C_{e}$ & 1127 & - & 1173 & 1403 & 1536 & 1608 \\
\cline { 3 - 8 } & $p$ & $-0,063$ & - & $-0,063$ & $-0,070$ & $-0,073$ & $-0,070$ \\
\hline \multirow{2}{*}{600} & $C_{e}$ & - & 1234 & - & 1812 & 3677 & - \\
\cline { 3 - 8 } & $p$ & - & $-0,098$ & - & $-0,122$ & $-0,194$ & - \\
\hline
\end{tabular}




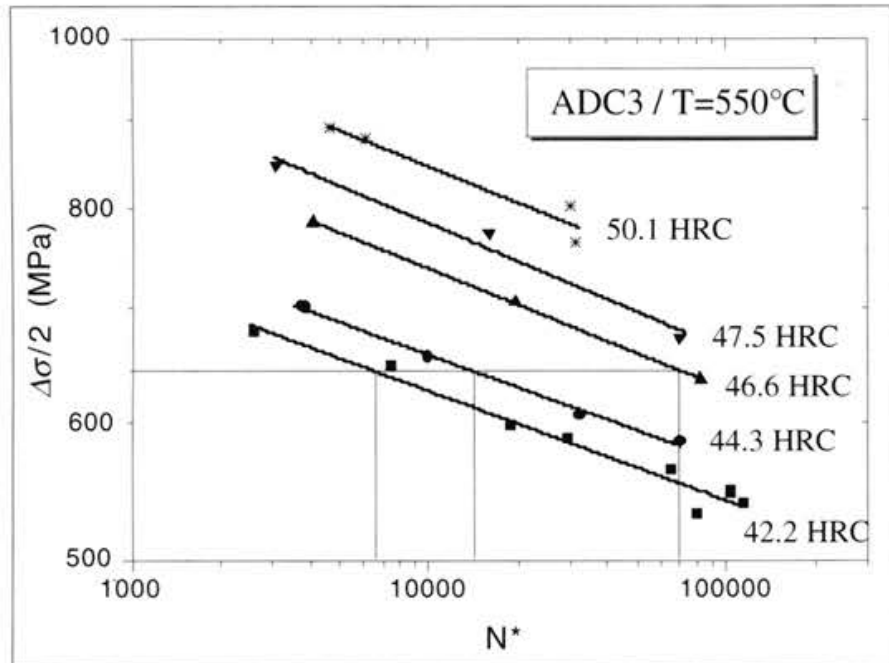

Fig. 14 - Diagramme de Basquin pour la nuance $\mathrm{ADC} 3$ à $550^{\circ} \mathrm{C}$ : effet du niveau de dureté.

Fig. 14 - Basquin diagram for the $A D C 3$ grade at $550^{\circ} \mathrm{C}$ : hardness effect.

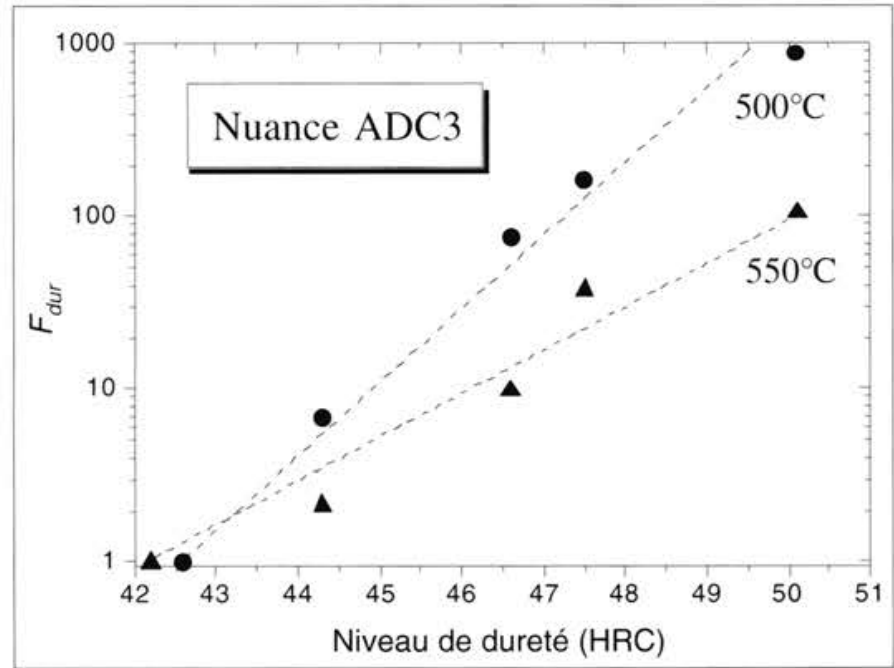

Fig. 15 - Rapport des durées de vie à amplitude de contrainte (à $N^{*} / 2$ ) équivalente.

Fig. 15 - Lifetimes ratio for a constant stress amplitude at $\mathrm{N} * / 2$.

élevés (i.e. $>47$ HRC) et donc les valeurs d'amplitudes de déformation plastique à demi-durée de vie sont relativement proches de celles relevées au premier cycle. Inversement, la déconsolidation de premier type étant plus élevée pour les niveaux de dureté faibles (i.e. $<47$ HRC), les amplitudes de déformation plastique augmentent considérablement, si bien que la droite de Manson-Coffin se trouve décalée vers le haut. La différence de résistance à la déformation plastique d'aciers possédant des niveaux de dureté différents pourrait être attribuée (au moins en partie) à une différence de mécanisme d'accommodation [22].

À $600^{\circ} \mathrm{C}$, les différences deviennent moins importantes car les écarts de résistance à la déformation plastique deviennent également plus faibles. En effet, la température du second revenu est égale, voire inférieure à la température d'essai. Le niveau de dureté relevé à température ambiante après le second revenu ne peut plus être considéré comme représentatif de la structure soumise à l'essai de fatigue isotherme.

Dans un diagramme de Basquin, les différences de durée de vie sont considérables (fig. 14). Jusqu'à $550^{\circ} \mathrm{C}$, les exposants de

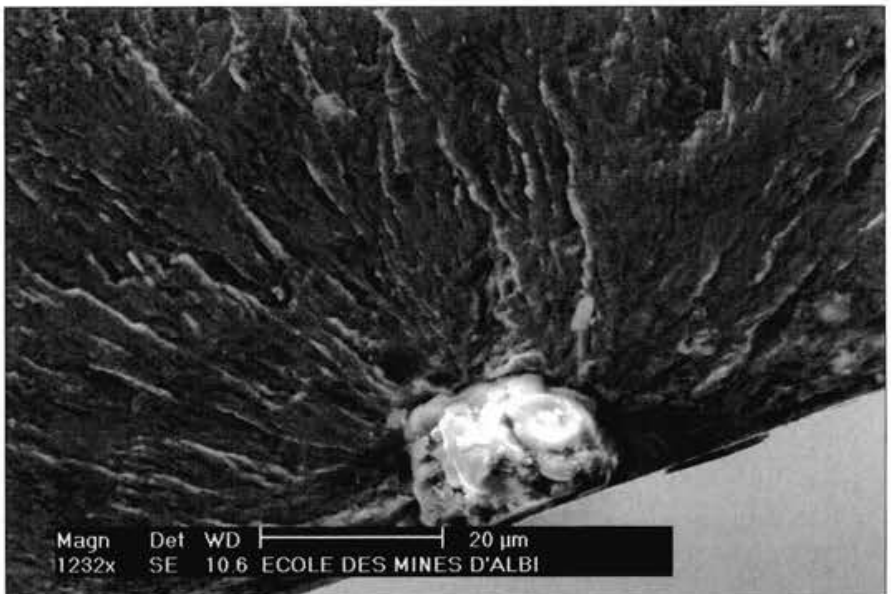

Photo 4 - Amorçage sur une NMI (nuance SMV3, T $=300^{\circ} \mathrm{C}, \mathrm{MEB}$ ).

Photo 4 - Crack initiation induced by a Non Metallic Inclusion (SMV3 grade, $T=300^{\circ} \mathrm{C}, S E M$ ).

Basquin ne dépendent que faiblement du niveau de dureté. Ces variations (moins de $25 \%$ ) seront négligées par la suite et les droites de Basquin seront considérées comme étant parallèles une à une ( $p$ constant). Un rapport de durée de vie noté $F_{d u r}$ pour une même amplitude de contrainte à demi-durée de vie peut être défini :

$$
F_{\text {dur }}=\frac{N^{*}{ }_{H R C_{2}}}{N^{*}{ }_{H R C_{1}}}=\left(\frac{C_{e_{H R C_{1}}}}{C_{e_{H R C_{2}}}}\right)^{\frac{1}{p}}
$$

La figure 15 présente l'évolution de $F_{d u r}$ en fonction du niveau de dureté à 500 et $550^{\circ} \mathrm{C}$ en prenant comme référence le niveau de dureté le plus faible. Le rapport de durée de vie pour une même amplitude de contrainte peut atteindre des valeurs proches de 1000 pour une augmentation de 8 points $\mathrm{HRC}$ à $500^{\circ} \mathrm{C}$, ce qui est considérable. En effet, les niveaux de dureté après revenus sont généralement obtenus avec une dispersion de 1 à 2 points HRC, ce qui correspond sur la figure 15 à un facteur de dispersion de 2 à 5 sur les durées de vie.

\section{Amorçage des fissures de fatigue}

\section{Principaux sites d'amorçage}

Les essais interrompus ont également montré que le stade d'amorçage occupe la majeure partie de la durée de vie lorsque les durées de vie sont supérieures à quelques dizaines de milliers de cycles. Puisque l'amorçage contrôle les durées de vie, nous avons observé systématiquement les sites d'amorçage sur les faciès de rupture et les surfaces extérieures après essai. Trois sites d'amorçage préférentiels ont été clairement identifiés:

- les inclusions non métalliques (non metallic inclusions: NMI) (photo 4);

- le joint de grain de la structure austénitique initiale (photo 5);

- le joint de latte (photo 6).

Les proportions de chaque cas $\rho^{\prime}, \rho^{\prime \prime}$ et $\rho^{\prime \prime}$ en fonction de la température sont indiquées sur la figure 16 où $\rho^{\prime}$ est la proportion d'amorçage sur NMI, $\rho$ " est la proportion d'amorçage aux joints de grain et $\rho$ "' est celle due à l'amorçage aux joints de latte.

Les mécanismes d'amorçage sont fortement dépendants d'une part de la température d'essai et d'autre part des effets 


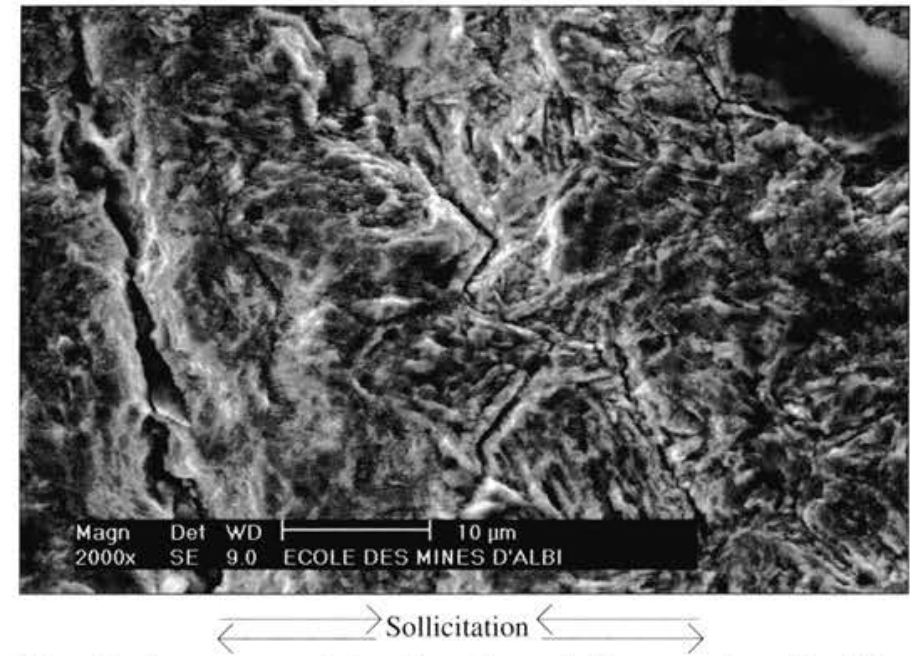

Photo 5 - Amorçage aux joints de grain austénitiques sur la surface libre (nuance $\mathrm{ADC} 3, \mathrm{~T}=600^{\circ} \mathrm{C}, \mathrm{MEB}$ ).

Photo 5 - Prior austenitic grain boundary crack initiation observed on the specimen surface $\left(A D C 3\right.$ grade, $T=600^{\circ} \mathrm{C}$. SEM).

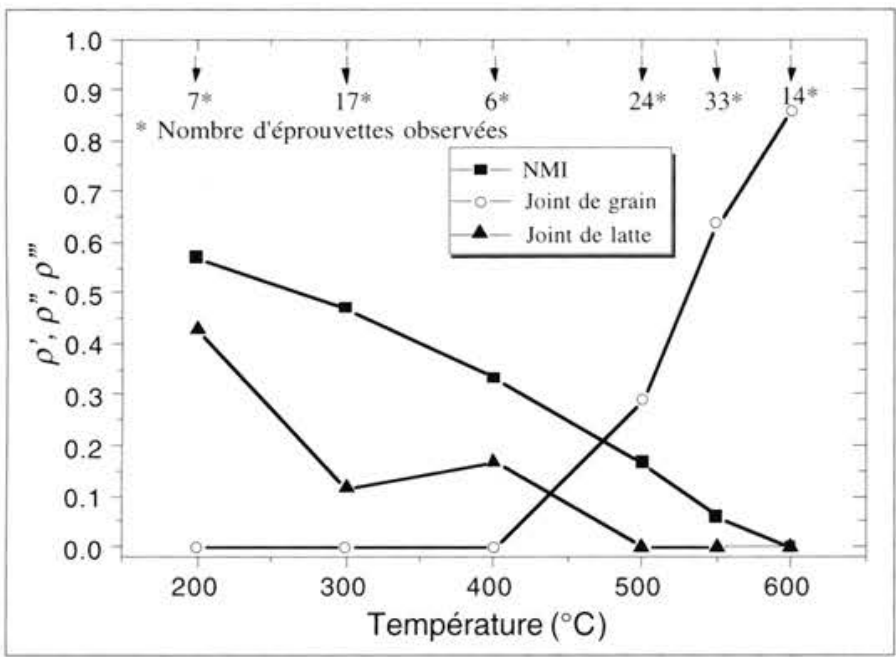

Fig. 16 - Proportion d'amorçage sur NMI, aux joints de grain et de latte en fonction de la température.

Fig.e 16 - Proportion of crack initiation observed on NMI, on grain and lath boundaries versus testing temperature.

de l'environnement. En effet, quelques essais réalisés sous atmosphère contrôlée (argon) lors d'une collaboration avec l'université de Braunschweig ont montré que l'amorçage aux joints de latte devient prépondérant aux températures élevées. Une perspective intéressante de ce travail est donc l'étude microscopique et quantitative des effets de l'oxydation qui semble jouer un rôle essentiel sur l'amorçage et la propagation des fissures [25]. Cette étude est en cours à l'Ecole des Mines d'Albi-Carmaux.

\section{Discussion sur l'amorçage sur inclusion}

En ce qui concerne l'amorçage sur inclusion, une observation au microscope optique sur une coupe en sens long permet de distinguer quatre types d'inclusions suivant leur morphologie. Quelle que soit la morphologie, on trouve majoritairement des NMI de type aluminate de chaux et silico-aluminate. Quelques sulfures présentant une morphologie allongée sont également détectés. Les quatre types d'inclusions sont les suivants:

- inclusion "globulaire" (i.e. facteur de forme $<1,5$ ) isolée;

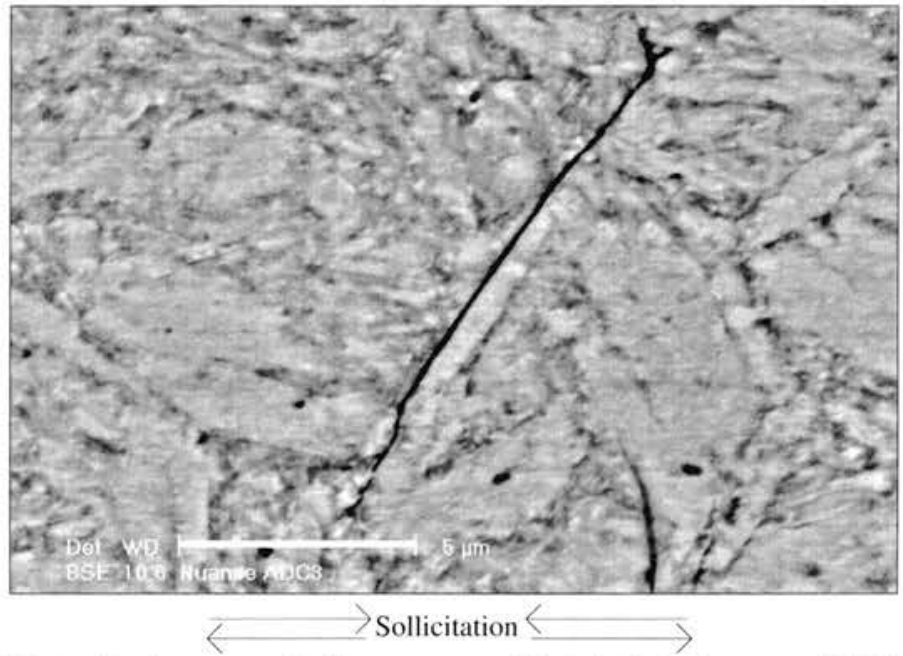

Photo 6 - Amorçage de fissure sur un joint de latte (nuance ADC3, $\left.\mathrm{T}=200^{\circ} \mathrm{C}, \mathrm{MEB}\right)$.

Photo 6 - Crack initiation observed on a martensitic lath boundary (ADC3 grade, $T=200^{\circ} \mathrm{C}, S E M$ ).

Fig. 17 .

Occurences

d'amorçage

sur NMI en

fonction de leur taille.

Fig. 17 . Number of crack initiations on $\mathrm{NMI}$ in relation to their size.

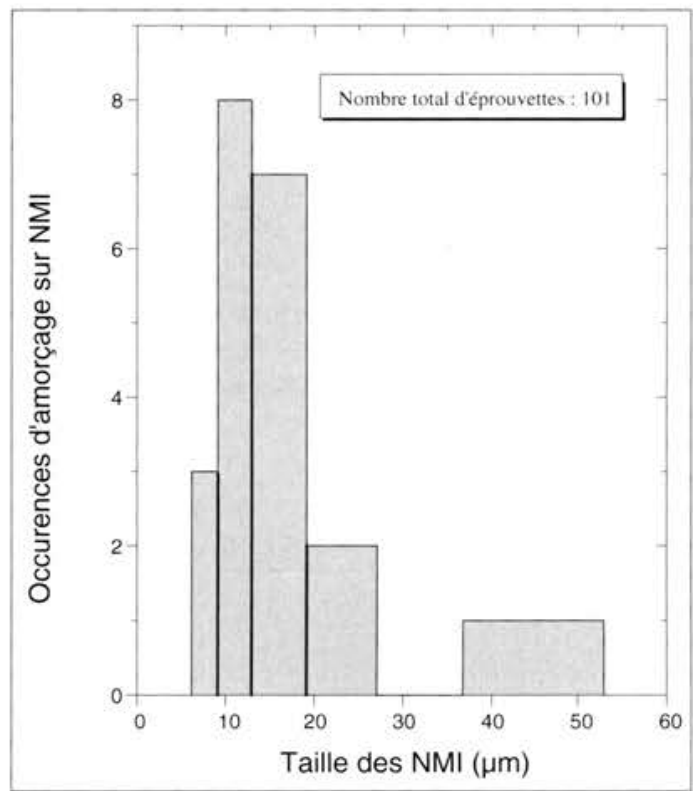

- inclusion "globulaire" en alignement;

- inclusion "allongée" pseudo-isolée (elles sont rarement totalement isolées);

- inclusion "allongée" en alignement.

Sur les faciès de rupture, la totalité des NMI ayant entraîné l'amorçage possède une morphologie globulaire ou anguleuse. L'histogramme de leur taille est donné sur la figure 17. Aucune NMI de forme allongée n'ayant été détectée, elles semblent donc moins nocives en ce qui concerne l'amorçage de fissures de fatigue. La constatation précédente se doit d'être nuancée par le positionnement des NMI par rapport à l'axe de sollicitation. En effet, quatre essais effectués en fatigue isotherme sur des éprouvettes prélevées en sens travers ont révélé un amorçage sur des NMI allongées ainsi que sur des alignements.

La figure 18 présente le diagramme de Basquin pour la nuance $\mathrm{SMV} 3$ à $300^{\circ} \mathrm{C}$, sur lequel nous avons indiqué les essais dont l'amorçage s'est produit sur une NMI. Pour une même amplitude de contrainte au cycle stabilisé, les durées de vie ne sont pas modifiées (du moins la perte de durée de vie est inférieure à la 


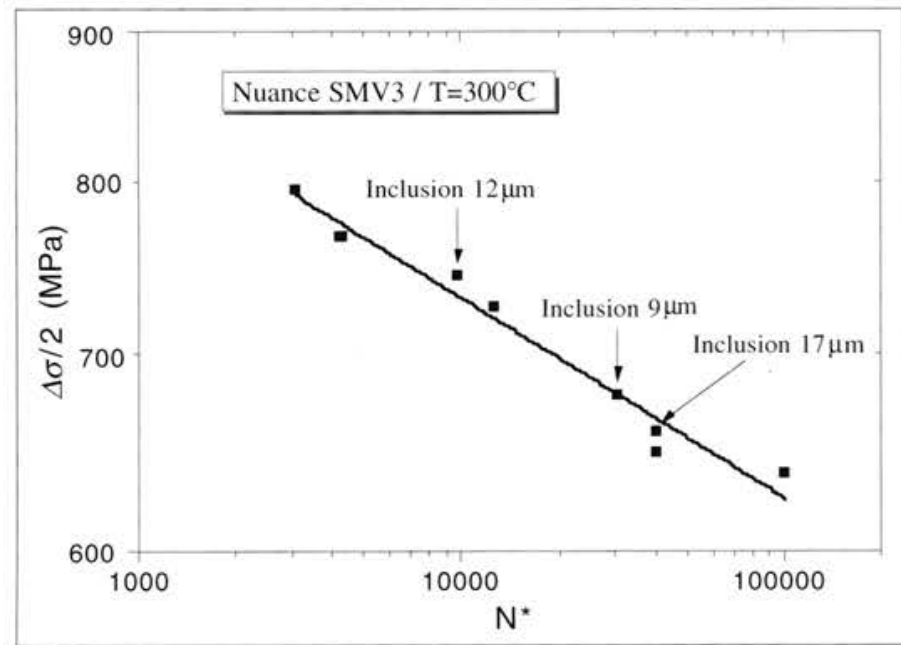

Fig. 18 - Diagramme de Basquin pour la nuance SMV3 $\left(300^{\circ} \mathrm{C}, 42 \mathrm{HRC}\right)$. Fig. 18 - Basquin diagram for the SMV 3 grade $\left(300^{\circ} \mathrm{C}, 42 \mathrm{HRC}\right)$.

\begin{tabular}{|c|c|c|c|}
\hline Classe $\boldsymbol{k}$ & $\begin{array}{c}\text { Diamètre } \\
(\mu \mathrm{m})\end{array}$ & $\begin{array}{c}\text { Quantité la plus faible } \\
\text { relevée dans une } \\
\text { plage de } 150 \mathrm{~mm}^{2}\end{array}$ & $\begin{array}{c}\text { Quantité la plus élevée } \\
\text { relevée dans } \\
\text { une plage de } 150 \mathrm{~mm}^{2}\end{array}$ \\
\hline 1 & $9-13$ & 70 & 135 \\
\hline 2 & $13-19$ & 24 & 51 \\
\hline 3 & $19-27$ & 3 & 17 \\
\hline 4 & $27-38$ & 0 & 6 \\
\hline 5 & $38-53$ & 0 & 4 \\
\hline
\end{tabular}

Tableau VIII - Distribution de la quantité d'inclusions en fonction de leur diamètre (six plages de $150 \mathrm{~mm}^{2}$ analysées).

Table VIII - Distribution of inclusions quantity in relation to their diameter (six $150 \mathrm{~mm}^{2}$ zones analysed).

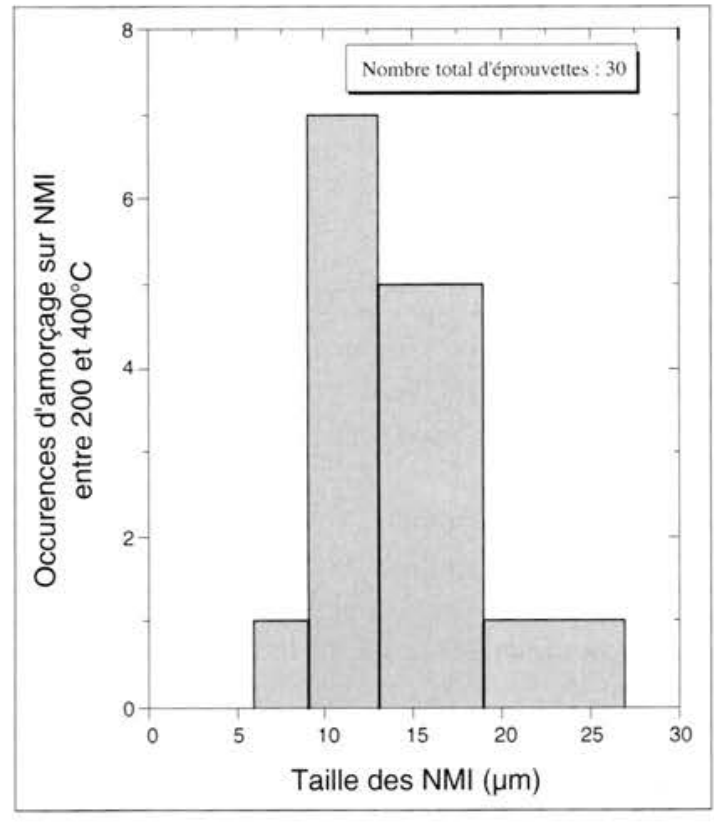

Fig. 19 Occurences d'amorçage sur NMI en fonction de leur taille $\left(200^{\circ} \mathrm{C} \leq T \leq\right.$ $\left.400^{\circ} \mathrm{C}\right)$.

Fig. 19 Number of crack initiations on $N M I$ in relation to their size $\left(200^{\circ} \mathrm{C} \leq T \leq\right.$ $400^{\circ} \mathrm{C}$ ).

Fig. 20 - Critère de nocivité des inclusions défini par A. De Bussac et al. [27]. Fig. 20 - Inclusions harmfulness criterion introduced by A. De Bussac [27].

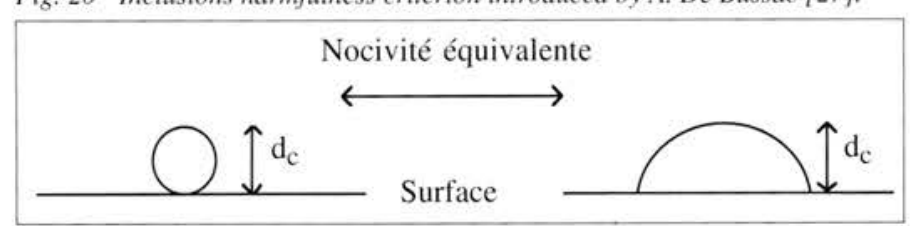

dispersion expérimentale) par l'amorçage sur une NMI. Les conclusions précédentes sont également valables pour une amplitude de déformation totale ou plastique à demi-durée de vie donnée. Cette constatation est de plus corroborée par l'observation microscopique, lors de multifissurations, de la présence de NMI sur le site d'amorçage de fissures secondaires alors que le site de la fissure principale était vierge. Les NMI $n$ 'induisent donc pas une perte de durée de vie mesurable.

A. Pineau [26] a proposé un modèle probabiliste permettant de prévoir pour une probabilité de rupture donnée, la taille du défaut qui est à l'origine de la rupture par fatigue. L'application de ce modèle nécessite la connaissance de l'histogramme de la taille des défauts. La distribution de NMI n'étant pas homogène, six plages de $150 \mathrm{~mm}^{2}$ ont été observées sur des échantillons différents. Le tableau VIII présente, pour chaque classe de taille, les valeurs la plus faible et la plus élevée du nombre de NMI relevées dans les six plages. Seules les NMI de morphologie globulaire (isolées ou en alignement) dont la taille est supérieure à $9 \mu \mathrm{m}$ dans le sens travers ont été comptées. La figure 17 montre que l'amorçage sur des inclusions de taille inférieure à $9 \mu \mathrm{m}$ est peu fréquent. La statistique d'amorçage sur NMI prise en compte concerne les 30 éprouvettes testées pour un niveau de dureté de $42 \mathrm{HRC}$ entre 200 et $400^{\circ} \mathrm{C}$, pour lesquelles 14 NMI situés en surface ont été observées sur les faciès de rupture. L'histogramme de la taille des NMI est donné sur la figure 19. Seule la plus petite NMI possède une morphologie anguleuse, les autres étant globulaires. Le modèle probabiliste adopté conduit à l'équation suivante [27]:

$$
p\left(d>d_{c}\right)=1-\exp \left\{-\sum_{D_{k}>D_{c}} N_{k} S\left(D_{k}-d_{c}^{k}\right)\right\}
$$

où $d_{c}$ est la profondeur critique d'une inclusion de surface (fig. 20), $p\left(d>d_{c}\right)$ est la probabilité de rupture à partir d'une inclusion de surface de profondeur supérieure à $d_{c}, S$ est la surface de la partie utile de l'échantillon; à chaque classe de taille $k$, on associe un diamètre des NMI $D_{k}$ et un nombre de NMI par unité de volume $N_{k} ; d_{c}^{k}$ est la profondeur critique pour les particules de diamètre $D_{k}$.

L'équation 7 est ensuite simplifiée par l'introduction d'un critère de nocivité en donnant une relation entre les différentes profondeurs critiques de chaque classe. A. De Bussac et al. [27] proposent le critère suivant: on suppose une même nocivité pour une même profondeur de pénétration : $d_{c}^{k}=d_{c}$.

L'équation 7 a la signification physique suivante: si des éprouvettes de même surface sont soumises à un chargement de fatigue, une proportion $1-p$ de ces éprouvettes devrait se rompre à partir d'une inclusion de surface de profondeur $d<d_{c}$ et une proportion $p$ devrait se rompre à partir d'une inclusion de surface de profondeur $d>d_{c}$.

Le calcul prenant en compte le critère de nocivité précédemment formulé donne pour une probabilité de rupture de 46,6\% $(14 / 30)$ une valeur de profondeur critique comprise entre $6 \mu \mathrm{m}$ et $10 \mu \mathrm{m}$. Cette fourchette de valeurs théoriques, bien que peu étroite, encadre de façon satisfaisante la valeur expérimentale puisque l'inclusion la plus petite observée sur le site d'amorçage possède une taille de $7,5 \mu \mathrm{m}$ (pour les 30 éprouvettes considérées: cf. fig. 19). L'application du modèle aux aciers à outils est cependant limitée compte tenu de l'hétérogénéité de la distribution des NMI. Si l'on admet que les NMI globulaires dont la taille est inférieure à la profondeur critique ne sont pas 
nocives (une fissure ne se propage pas à partir de l'inclusion), la proportion d'amorçage sur inclusion peut s'expliquer par la probabilité de présence d'une NMI globulaire de taille supérieure à la profondeur critique en surface.

\section{Conclusion}

Le travail réalisé est une contribution à l'étude du comportement et de l'endommagement cycliques des aciers martensitiques revenus, jusqu'à présent peu explorés.

- Nous avons mis en évidence l'instabilité cyclique continue de l'acier X 38 CrMoV 5. Bien que les écarts d'amplitude de contrainte entre le premier cycle et le cycle $N^{*}$ dépendent fortement des conditions de sollicitation et de la résistance du matériau, l'instabilité est toujours constatée y compris pour des durées de vie supérieures à $10^{5}$ cycles [22].

- Le comportement cyclique peut être décomposé en deux stades:

- un stade de variation de l'amplitude de contrainte en loi puissance qui peut atteindre $50 \%$ de la durée de vie. Nous obtenons une déconsolidation cyclique dont l'amplitude est une fonction croissante de l'amplitude de sollicitation et décroissante de la température d'essai et du niveau de dureté. Pour des niveaux de dureté supérieurs à $47 \mathrm{HRC}$, une consolidation cyclique est obtenue pour les faibles amplitudes de sollicitation:

- un stade de déconsolidation linéaire où l'amplitude de déconsolidation par cycle est proportionnelle à l'amplitude de déformation plastique à demi-durée de vie.
Dans le domaine oligocyclique, les déconsolidations peuvent atteindre $25 \%$ de l'amplitude de contrainte au premier cycle. Elles doivent donc être prises en compte lors d'un calcul de dimensionnement d'un outillage fortement sollicité.

Etablir une corrélation entre une mesure de dureté relevée à température ambiante et une température de fonctionnement d'un outillage lors d'une expertise n'est donc pas une entreprise triviale. Non seulement une perte de dureté constatée à froid ne témoigne pas de sa valeur à température élevée, mais elle englobe l'adoucissement d'origine thermique et mécanique. Un adoucissement relevé sur un outillage n'est donc pas synonyme de "surchauffe", mais peut être dû à une plastification cyclique.

- Nous avons étudié les influences de la température et du niveau de dureté sur les durées de vie. À amplitude de déformation plastique équivalente, les durées de vie sont peu modifiées, à l'exception des températures et des niveaux de dureté élevés $\left(600^{\circ} \mathrm{C}\right.$ et $\left.50 \mathrm{HRC}\right)$. À amplitude de contrainte équivalente, une augmentation de la température ou une diminution du niveau de dureté induisent des pertes de durée de vie considérables.

- Trois sites d'amorçage préférentiels ont été mis en évidence: Les NMI et le joint de latte aux basses températures (200$400^{\circ} \mathrm{C}$ ) et le joint de grain de la structure austénitique initiale aux températures élevées $\left(500-600^{\circ} \mathrm{C}\right)$.

\section{RÉFÉRENCES}

[1] C. Bournicon, "Sollicitations et modes d'endommagement des outillages de forge à chaud", Traitement Thermique, 246, 1991, pp. 70-77.

[2] J.Å. GAVEN, "Tool performance of aluminium die-casting dies", Proceedings of the conference "Tools for die casting", Sunne, Sweden, 26-28 sept 1983, edited by Uddeholm and the Swedish Institute for Metals Research, Tryckeri AB Dahlberg \& Co, 1983, pp. 267-289.

[3] G. Primon, J. Beguinot, L. Cadiou, "Contribution à la mise au point du traitement thermique de recuit sur acier à outils pour travail à chaud de type Z $38 \mathrm{CDV} 5$ (AISI H11)", Traitement Thermique, 217, 1988, pp. 13-15.

[4] M. Siaut, "Evolution du choix des aciers spéciaux destinés à la fabrication d'empreintes pour injection d'alliages légers", Rapport interne Aubert \& Duval, 1994.

[5] Norme Française NF A 04-102, "Produits sidérurgiques: détermination de la grosseur du grain ferritique ou austénitique des aciers", AFNOR, novembre 1980.

[6] M. Siaut, "Traitement thermique des aciers Z 38 CDV 5 pour moules sous pression d'alliages légers et optimisation des propriétés d'emploi", Traitement Thermique, 217, 1988, pp. 21-26.

[7] Z. HU, J. XIAO, "Cyclic softening characteristics and mechanism of hot work die steels during low-cycle fatigue", in "Fatigue 90", Proceedings of the $4^{\text {th }}$ international conference on fatigue and fatigue thresholds, Honolulu, 1990, pp. 469-474.

[8] J.C. Earthman, G. Eggeler, B. Ilschner, "Deformation and damage processes in a $12 \% \mathrm{Cr}-\mathrm{Mo}-\mathrm{V}$ steel under high temperature low cycle fatigue conditions in air and vacuum", Materials Science and Engineering, vol. A 110, 1989, pp. 103-114.

[9] F. Martin, J. Petit, J. DE Fouquet, "Cyclic behaviour of a medium carbon micro-alloyed steel", Materials Science and Engineering, vol. 61, 1983, pp. 237-246.

[10] R.A. Fournelle, E.A. Grey, M.E. Fine, "Fatigue behavior of a precipitation hardening Ni-Al-Cu medium carbon steel", Metallurgical Transactions, vol. 7A, 1976, pp. 669-682.

[11] M.C. Mataya, R.A. Fournelle, "Fatigue behaviour of a NiAl precipitation hardening medium carbon steel", Metallurgical Transactions, vol. 9A, 1978, pp. 917-925.

[12] S.I. Kwun, R.A. Fournelle, "Low cycle fatigue behavior of a quenched and tempered niobium bearing HSLA steel", Metallurgical Transactions, vol. 11A, 1980, pp. 1429-1437.

[13] K. Kanazawa, K. Yamaguchi, K. Kobayashi, "The temperature dependance of low cycle fatigue behaviour of martensitic stainless steels", Materials Science and Engineering, vol. 40, 1979, pp. 89-96.

[14] J.B. Vogt, G. Degallaix, J. Foct, "Cyclic mechanical behavior and microstructure of a $12 \mathrm{Cr}-\mathrm{Mo}-\mathrm{V}$ martensitic stainless steel, Fatigue \& fracture of Engineering Materials and Structures, vol. 11, n 6, 1988, pp. 435-446.

[15] J.B. VoGt, C. BIGEON, J. Foct, "Combined effect of nitrogen and silicon on low cycle fatigue of $12 \% \mathrm{Cr}$ martensitic stainless steels", Zeitschrift für Metallkunde, vol. 85, n², 1994, pp. $92-99$.

[16] S.R. Mediratta, V. Ramaswamy, V. Singh, R. Rao, "Cyclic work hardening and formation of dislocation substructures during low-cycle fatigue of tempered dual-phase steels", Steel Research, vol. 61, n 7, 1990, pp. 325-332.

17] H.J. Christ, C. Sommer, H. Mughrabi, A.P. Voskamp, J.M. Beswick, F. HENGERER, "Fatigue behaviour of three variants of the roller bearing steel SAE 52100", Fatigue \& fracture of Engineering Materials and Structures, vol. 15, 9, 1992, pp. 855-870. 
[18] H.J. Chang, J.J. Kal, C.H. Tsal, "Cyclic deformation property and microstructure study of HT-9 ferritic steel at elevated temperatures", Journal of Nuclear Materials, vol. 212, 215, 1994. pp. $574-578$.

[19] J.H. Beatty, G.J. Shiflet, "Strain distribution effects on the lowcycle fatigue of Fe-C-Mo steels", Metallurgical Transactions A, Physical metallurgy and Materials Science, vol. 22, n 3, 1991, pp. 675-683.

[20] H.J. ChanG, C.H. TSAI, J.J. Kal, "Effects of temperature on the cyclic deformation behaviour and microstructural changes of 12Cr-1MoVW martensitic stainless steel", International Journal of Pressure Vessels and Piping, vol. 59, 1-3, 1994, pp. 31-40.

[21] A. Joarder, N.S. Cheruvu, D.S. Sarma, "Influence of high temperature low cycle fatigue deformation on the microstructure of a CrMoV rotor steel subjected to long-term service exposure at $425^{\circ} \mathrm{C}$ and retempering at $677^{\circ} \mathrm{C} "$, Materials Characterization, vol. 28, n०4, 1992, pp. 121-131.

[22] D. Delagnes, "Comportement et tenue en fatigue isotherme d'aciers à outils Z 38 CDV 5 autour de la transition fatigue oligocyclique-endurance," Thèse de doctorat de l'Ecole des Mines de
Paris, spécialité: Science et Génie des Matériaux, mars 1998.

[23] Z.G. WANG, K. RahKa, P. Nenonen, C. Laird, "Changes in morphology and composition of carbides during cyclic deformation at room and elevated temperature and their effect on mechanical properties of Cr-Mo-V steel”, Acta Metallurgica, vol. 33, n' ${ }^{\circ}$, 1985, pp. 2129-2141.

[24] S.R. Mediratta, V. Ramaswamy, V. Singh, "Evolution of dislocation substructures during strain cycling of a dual phase steel", Transactions of the Indian Institute of Metals, vol. 43, 1990, pp. 300-302.

[25] N. Tsujil, G. Abe, K. Fukaura, H. Sunada, "Effect of testing atmosphere on low cycle fatigue of hot work tool steel at elevated temperature", ISIJ International, vol. 35, n 7, 1995, pp. 920-926.

[26] A. Pineau, "Superalloy discs durability and damage tolerance in relation to inclusions," in High temperature materials for power engineering, éditions E. Bachelet, 1990, pp. 913-934.

[27] A. De Bussac, J.C. Lautridou, "La prévision de l'amorçage en surface de fissures de fatigue dans les alliages MDP : Un modèle probabiliste", La Revue Scientifique SNECMA, 5, 1994, pp. 63-71. 